\title{
How frequently is a system of 2-linear Boolean equations solvable?
}

\author{
Boris Pittel* and Ji-A Yeum \\ Ohio State University, Columbus, Ohio, USA \\ bgp@math.ohio-state.edu, yeum@math.ohio-state.edu
}

Submitted: Sep 7, 2009; Accepted: Jun 19, 2010; Published: Jun 29, 2010

Mathematics Subject Classifications: 05C80, 05C30, 34E05, 60C05

\begin{abstract}
We consider a random system of equations $x_{i}+x_{j}=b_{(i, j)}(\bmod 2),\left(x_{u} \in\right.$ $\left.\{0,1\}, b_{(u, v)}=b_{(v, u)} \in\{0,1\}\right)$, with the pairs $(i, j)$ from $E$, a symmetric subset of $[n] \times[n]$. $E$ is chosen uniformly at random among all such subsets of a given cardinality $m$; alternatively $(i, j) \in E$ with a given probability $p$, independently of all other pairs. Also, given $E, \operatorname{Pr}\left\{b_{e}=0\right\}=\operatorname{Pr}\left\{b_{e}=1\right\}$ for each $e \in E$, independently of all other $b_{e^{\prime}}$. It is well known that, as $m$ passes through $n / 2$ ( $p$ passes through $1 / n$, resp. $)$, the underlying random graph $G(n$, \# edges $=m),(G(n, \operatorname{Pr}($ edge $)=p)$, resp.) undergoes a rapid transition, from essentially a forest of many small trees to a graph with one large, multicyclic, component in a sea of small tree components. We should expect then that the solvability probability decreases precipitously in the vicinity of $m \sim n / 2(p \sim 1 / n)$, and indeed this probability is of order $(1-2 m / n)^{1 / 4}$, for $m<n / 2\left((1-p n)^{1 / 4}\right.$, for $p<1 / n$, resp.). We show that in a near-critical phase $m=(n / 2)\left(1+\lambda n^{-1 / 3}\right)\left(p=\left(1+\lambda n^{-1 / 3}\right) / n\right.$, resp. $), \lambda=o\left(n^{1 / 12}\right)$, the system is solvable with probability asymptotic to $c(\lambda) n^{-1 / 12}$, for some explicit function $c(\lambda)>0$. Mike Molloy noticed that the Boolean system with $b_{e} \equiv 1$ is solvable iff the underlying graph is 2-colorable, and asked whether this connection might be used to determine an order of probability of 2-colorability in the near-critical case. We answer Molloy's question affirmatively and show that, for $\lambda=o\left(n^{1 / 12}\right)$, the probability of 2 -colorability is $\lesssim 2^{-1 / 4} e^{1 / 8} c(\lambda) n^{-1 / 12}$, and asymptotic to $2^{-1 / 4} e^{1 / 8} c(\lambda) n^{-1 / 12}$ at a critical phase $\lambda=O(1)$, and for $\lambda \rightarrow-\infty$.
\end{abstract}

\section{Introduction}

A system of 2-linear equations over $G F(2)$ with $n$ Boolean variables $x_{1}, \ldots, x_{n} \in\{0,1\}$ is

$$
x_{i}+x_{j}=b_{i, j}(\bmod 2), \quad b_{i, j}=b_{j, i} \in\{0,1\} ; \quad(i \neq j) .
$$

*Pittel's research supported in part by NSF Grants DMS-0406024, DMS-0805996 
Here the unordered pairs $(i, j)$ correspond to the edge set of a given graph $G$ on the vertex set $[n]$. The system (1.1) certainly has a solution when $G$ is a tree. It can be obtained by picking an arbitrary $x_{i} \in\{0,1\}$ at a root $i$ and determining the other $x_{j}$ recursively along the paths leading away from the root. There is, of course, a twin solution $\bar{x}_{j}=1-x_{j}, j \in[n]$. Suppose $G$ is not a tree, i.e. $\ell(G):=e(G)-v(G) \geqslant 0$. If $T$ is a tree spanning $G$, then each of additional edges $e_{1}, \ldots, e_{\ell(G)+1}$ forms, together with the edges of $T$, a single cycle $C_{t}, t \leqslant \ell(G)+1$. Obviously, a solution $x_{j}(T)$ of a subsystem of (1.0.1) induced by the edges of $T$ is a solution of (1.0.1) provided that

$$
b_{i, j}=x_{i}(T)+x_{j}(T),(i, j)=e_{1}, \ldots, e_{\ell(G)+1} ;
$$

equivalently

$$
\sum_{e \in E\left(C_{t}\right)} b_{e}=0(\bmod 2), \quad t=1, \ldots, \ell(G)+1 .
$$

So, intuitively, the more edges $G$ has the less likely it is that the system (1.0.1) has a solution. We will denote the number of solutions by $S(G)$.

In this paper we consider solvability of a random system (1.0.1). Namely $G$ is either the Bernoulli random graph $G(n, p)=G(n, \operatorname{Pr}($ edge $)=p)$, or the Erdős-Rényi random graph $G(n, m)=G(n, \#$ of edges $=m)$. Further, conditioned on the edge set $E(G(n, p))$ $\left(E(G(n, m)\right.$ resp. $), b_{e}$ 's are independent, and $\operatorname{Pr}\left(b_{e}=1\right)=\hat{p}$, for all $e$. We focus on $\hat{p}=1 / 2$ and $\hat{p}=1 . \hat{p}=1 / 2$ is the case when $b_{e}$ 's are "absolutely random". For $\hat{p}=1, b_{e}$ 's are all ones. Mike Molloy [19], who brought this case to our attention, noticed that here (1.0.1) has a solution iff the underlying graph is bipartite, 2-colorable in other words.

It is well known that, as $\mathrm{m}$ passes through $n / 2$ ( $p$ passes through $1 / n$, resp.), the underlying random graph $G(n, m),(G(n, p)$, resp.) undergoes a rapid transition, from essentially a forest of many small trees to a graph with one large, multicyclic, component in a sea of small tree components. Bollobás [4], [5] discovered that, for $G(n, m)$, the phase transition window is within $\left[m_{1}, m_{2}\right]$, where

$$
m_{1,2}=n / 2 \pm \lambda n^{2 / 3}, \quad \lambda=\Theta\left(\ln ^{1 / 2} n\right) .
$$

Euczak [15] was able to show that the window is precisely $\left[m_{1}, m_{2}\right]$ with $\lambda \rightarrow \infty$ however slowly. (See Łuczak et al [17] for the distributional results on the critical graphs $G(n, m)$ and $G(n, p)$.) We should expect then that the solvability probability decreases precipitously for $m$ close to $n / 2$ ( $p$ close to $1 / n$ resp.). Indeed, for a multigraph version of $G(n, m)$, Kolchin [14] proved that this probability is asymptotic to

$$
\frac{(1-\gamma)^{1 / 4}}{(1-(1-2 \hat{p}) \gamma)^{1 / 4}}, \quad \gamma:=\frac{2 m}{n},
$$

if $\lim \sup \gamma<1$. See Creignon and Daudé [9] for a similar result. Using the results from Pittel [21], we show (see Appendix) that for the random graphs $G(n, \gamma n / 2)$ and $G(n, p=\gamma / n)$, with $\lim \sup \gamma<1$, the corresponding probability is asymptotic to

$$
\frac{(1-\gamma)^{1 / 4}}{(1-(1-2 \hat{p}) \gamma)^{1 / 4}} \exp \left[\frac{\gamma}{2} \hat{p}+\frac{\gamma^{2}}{2} \hat{p}(1-\hat{p})\right]
$$


The relations (1.0.4), (1.0.5) make it plausible that, in the nearcritical phase $|m-n / 2|=$ $O\left(n^{2 / 3}\right)$, the solvability probability is of order $n^{-1 / 12}$. Our goal is to confirm, rigorously, this conjecture.

To formulate our main result, we need some notations. Let $\left\{f_{r}\right\}_{r} \geqslant 0$ be a sequence defined by an implicit recurrence

$$
f_{0}=1, \quad \sum_{k=0}^{r} f_{k} f_{r-k}=\varepsilon_{r}, \quad \varepsilon_{r}:=\frac{(6 r) !}{2^{5 r} 3^{2 r}(3 r) !(2 r) !} .
$$

Equivalently, the formal series $\sum_{r} x^{r} f_{r}, \sum_{r} x^{r} \varepsilon_{r}$ (divergent for all $x \neq 0$ ) satisfy

$$
\left(\sum_{r} x^{r} f_{r}\right)^{2}=\sum_{r} x^{r} \varepsilon_{r}
$$

It is not difficult to show that

$$
\frac{\varepsilon_{r}}{2}\left(1-\frac{1}{r}\right) \leqslant f_{r} \leqslant \frac{\varepsilon_{r}}{2}, \quad r>0 .
$$

For $y, \lambda \in \mathbb{R}$, let $A(y, \lambda)$ denote the sum of a convergent series,

$$
A(y, \lambda)=\frac{e^{-\lambda^{3} / 6}}{3^{(y+1) / 3}} \sum_{k \geqslant 0} \frac{\left(\frac{1}{2} 3^{2 / 3} \lambda\right)^{k}}{k ! \Gamma[(y+1-2 k) / 3]} .
$$

We will write $B_{n} \sim C_{n}$ if $\lim _{n \rightarrow \infty} B_{n} / C_{n}=1$, and $B_{n} \lesssim C_{n}$ if $\limsup _{n} B_{n} / C_{n} \leqslant 1$. Let $S_{n}$ denote the random number of solutions (1.0.1) with the underlying graph being either $G(n, m)$ or $G(n, p)$, i. e. $S_{n}=S(G(n, m))$ or $S_{n}=S(G(n, p))$, and the (conditional) probability of $b_{e}=1$ for $e \in E(G(n, m))(e \in E(G(n, p))$ resp.) being equal $\hat{p}$.

Theorem 1.1. (i) Let $\hat{p}=1 / 2$. Suppose that

$$
m=\frac{n}{2}\left(1+\lambda n^{-1 / 3}\right), \quad p=\frac{1+\lambda n^{-1 / 3}}{n}, \quad|\lambda|=o\left(n^{1 / 12}\right) .
$$

Then, for both $G(n, m)$ and $G(n, p)$,

$$
\operatorname{Pr}\left(S_{n}>0\right) \sim n^{-1 / 12} c(\lambda)
$$

where

$$
c(\lambda):= \begin{cases}e^{3 / 8}(2 \pi)^{1 / 2} \sum_{r \geqslant 0} \frac{f_{r}}{2^{r}} A(1 / 4+3 r, \lambda), & \lambda \in(-\infty, \infty) ; \\ e^{3 / 8}|\lambda|^{1 / 4}, & \lambda \rightarrow-\infty ; \\ \frac{e^{3 / 8}}{2 \cdot 3^{3 / 4}} \lambda^{1 / 4} \exp \left(-4 \lambda^{3} / 27\right), & \lambda \rightarrow \infty .\end{cases}
$$

(ii) Let $\hat{p}=1$. Then, with $c(\lambda)$ replaced by $c_{1}(\lambda):=2^{-1 / 4} e^{1 / 8} c(\lambda),(1.0 .11)$ holds for both $G(n, m)$ and $G(n, p)$ if either $\lambda=O(1)$, or $\lambda \rightarrow-\infty,|\lambda|=o\left(n^{1 / 12}\right)$. For $\lambda \rightarrow \infty$, $\lambda=o\left(n^{1 / 12}\right)$,

$$
\operatorname{Pr}\left(S_{n}>0\right) \lesssim n^{-1 / 12} c_{1}(\lambda)
$$


Notes. 1. For $G(n, m)$ with $\lambda \rightarrow-\infty$, and $\hat{p}=1 / 2$, our result blends, qualitatively, with the estimate (1.0.4) from [14] and [9] for a subcritical multigraph, and becomes the estimate (1.0.5) for the subcritical graphs $G(n, m)$ and $G(n, p)$.

2. The part (ii) answers Molloy's question: the critical graph $G(n, m)(G(n, p)$ resp.) is bichromatic (bipartite) with probability $\sim c_{1}(\lambda) n^{-1 / 12}$.

Very interestingly, the largest bipartite subgraph of the critical $G(n, p)$ can be found in expected time $O(n)$, see Coppersmith et al [8], Scott and Sorkin [23] and references therein. The case $\lambda \rightarrow \infty$ of (ii) strongly suggests that the supercritical graph $G(n, p=c / n)$, $(G(n, m=c n / 2)$ resp. $)$, i. e. with $\liminf c>1$, is bichromatic with exponentially small probability. In [8] this exponential smallness was established for the conditional probability, given that the random graph has a giant component.

Here is a technical reason why, for $\lambda=O(1)$ at least, the asymptotic probability of 2-colorability is the asymptotic solvability probability for (1.0.1) with $\hat{p}=1 / 2$ times $2^{-1 / 4} e^{1 / 8}$. Let $C_{\ell}(x)\left(C_{\ell}^{e}(x)\right.$ resp.) denote the exponential generating functions of connected graphs $G$ (graphs $G$ without odd cycles resp.) with excess $e(G)-v(G)=\ell \geqslant 0$. It turns out that, for $|x|<e^{-1}$ (convergence radius of $C_{\ell}(x), C_{\ell}^{e}(x)$ ), and $x \rightarrow e^{-1}$,

$$
C_{\ell}^{e}(x) \begin{cases}\sim \frac{1}{2^{\ell+1}} C_{\ell}(x), & \ell>0, \\ =\frac{1}{2} C_{0}(x)+\ln \left(2^{-1 / 4} e^{1 / 8}\right)+o(1), & \ell=0 .\end{cases}
$$

Asymptotically, within the factor $e^{\ln \left(2^{-1 / 4} e^{1 / 8}\right)}$, this reduces the problem to that for $\hat{p}=$ $1 / 2$. Based on (1.0.5), we conjecture that generally, for $\hat{p} \in(0,1]$, and the critical $p$, $\operatorname{Pr}\left(S_{n}>0\right)$ is that probability for $\hat{p}=1 / 2$ times

$$
(2 \hat{p})^{-1 / 4} \exp \left[-\frac{(1-\hat{p})^{2}}{2}+\frac{1}{8}\right] .
$$

(For $\hat{p}=0, \operatorname{Pr}\left(S_{n}>0\right)=1$ obviously.)

3. While working on this project, we became aware of a recent paper [10] by Daudé and Ravelomanana. They studied a close but different case, when a system of $m$ equations is chosen uniformly at random among all $n(n-1)$ equations of the form (1.0.1). In particular, it is possible to have pairs of clearly contradictory equations, $x_{i}+x_{j}=0$ and $x_{i}+x_{j}=1$. For $m=O(n)$ the probability that none of these simplest contradictions occurs is bounded away from zero. So, intuitively, the system they studied is close to ours with $G=G(n, m)$ and $\hat{p}=1 / 2$. Our asymptotic formula (1.0.11), with two first equations in (1.0.12), in this case is similar to Daudé-Ravelomanana's main theorem, but there are some puzzling differences. The exponent series in their equation (2) is certainly misplaced; their claim does not contain our sequence $\left\{f_{r}\right\}$.

As far as we can judge by a proof outline in [10], our argument is quite different. Still, like [10], our analysis is based on the generating functions of sparse graphs discovered, to a great extent, by Wright [25], [26]. We gratefully credit Daudé and Ravelomanana for 
stressing importance of Wright's bounds for the generating function $C_{\ell}(x)$. These bounds play a substantial role in our argument as well.

4. We should mention a large body of work on a related, better known, $2-S A T$ problem, see for instance Bollobás et al [6], and references therein. It is a problem of existence of a truth-satisfying assignment for the variables in the conjunction of $m$ random disjunctive clauses of a form $x_{i} \vee x_{j},(i, j \in[n])$. It is well known, Chvátal and Reed [7], that the existence threshold is $m / n=1$. It was proved in [6] that the phase transition window is $\left[m_{1}, m_{2}\right]$, with

$$
m_{1,2} \pm \lambda n^{2 / 3}, \quad|\lambda| \rightarrow \infty \text { however slowly }
$$

and that the solvability probability is bounded away from both 0 and 1 iff $m+O\left(n^{2 / 3}\right)$.

5. A natural extension of the system (1.0.1) is a system of $k$-linear equations

$$
\sum_{i \in e} x_{i}=b_{e}(\bmod 2)
$$

where $e$ runs over a set $E$ of (hyper)edges of a $k$-uniform hypergraph $G, k \geqslant 2$, on the vertex set $[n]$, Kolchin [14]. Suppose $G$ is chosen uniformly at random among all $k$ uniform graphs with a given number $m$ of edges, and, given $G$, the $b_{e}$ 's are independent Bernoullis. Dubois and Mandler [11] showed that, for $k=3, m / n=0.91793 \ldots$ is a sharp threshold for the limiting solvability probability.

The paper is organized as follows.

In the section 2 we work on the $G(n, p)$ and $\hat{p}=1 / 2$ case. Specifically in the (sub)section 2.1 we express the solvability probability, $\operatorname{Pr}\left(S_{n}>0\right)$, and its truncated version, as a coefficient by $x^{n}$ in a power series based on the generating functions of the sparsely edged (connected) graphs. We also establish positive correlation between solvability and boundedness of a maximal "excess", and determine a proper truncation of the latter dependent upon the behavior of $\lambda$. In the section (2.2) we provide a necessary information about the generating functions and their truncated versions involved in the formula and the bounds for $\operatorname{Pr}\left(S_{n}>0\right)$. In the section 2.3 we apply complex analysis techniques to the "coefficient by $x^{n}$ " formulas and obtain a sharp asymptotic estimate for $\operatorname{Pr}\left(S_{n}>0\right)$ for $|\lambda|=o\left(n^{1 / 12}\right)$.

In the section 3 we transfer the results of the section 2 to the $G(n, m)$ and $\hat{p}=1 / 2$ case .

In the section 4 we establish the counterparts of the results from the sections 2,3 for $G(n, p), G(n, m)$ with $\hat{p}=1$. An enumerative ingredient of the argument is an analogue of Wright's formulas for the generating functions of the connected graphs without odd cycles.

In Appendix we prove some auxilliary technical results, and an asymptotic formula for $\operatorname{Pr}\left(S_{n}>0\right)$ in the subcritical case, i. e. when the average vertex degree is less than, and bounded away from 1 . 


\section{Solvability probability: $G(n, p)$ and $\hat{p}=1 / 2$.}

\subsection{Representing bounds for $\operatorname{Pr}\left(S_{n}>0\right)$ as a coefficient of $x^{n}$ in a power series.}

Our first step is to compute the probability of the event $\left\{S_{n}>0\right\}$, conditioned on $G(n, p)$. Given a graph $G=(V(G), E(G))$, we denote $v(G)=|V(G)|, e(G)=|E(G)|$.

Lemma 2.1. Given a graph $G$ on $[n]$, let $c(G)$ denote the total number of its components $H_{i}$. Then

$$
\begin{aligned}
\operatorname{Pr}\left(S_{n}>0 \mid G(n, p)=G\right) & =\prod_{i=1}^{c(G)}\left(\frac{1}{2}\right)^{e\left(H_{i}\right)-\left(v\left(H_{i}\right)-1\right)} \\
& =\left(\frac{1}{2}\right)^{X(G)}, \quad X(G):=e(G)-n+c(G) .
\end{aligned}
$$

Consequently

$$
\operatorname{Pr}\left(S_{n}>0\right)=\mathbf{E}\left[\left(\frac{1}{2}\right)^{X(G(n, p))}\right] .
$$

Proof of Lemma 2.1. Recall that, conditioned on $G(n, p)$, the edge variables $b_{e}$ 's are mutually independent. So it is suffices to show that a system (1.0.1) for a connected graph $H$, with independent $b_{e}, e \in E(H)$, such that $\operatorname{Pr}\left(b_{e}=1\right)=1 / 2$, is solvable with probability $(1 / 2)^{\ell+1}$, where $\ell=e(H)-v(H)$.

Let $T$ be a tree spanning $H$. Let $\boldsymbol{x}(T):=\left\{x_{i}(T)\right\}_{i \in V(H)}$ be the solution of the subsystem of (1.0.1) corresponding to $v(H)-1$ edges of $T$, with $x_{i_{0}}=1$ say, for a specified "root" $i_{0} \cdot \boldsymbol{x}(T)$ is a solution of the whole system (1.0.1) iff

$$
b_{e}=x_{i}(T)+x_{j}(T), \quad((i, j)=e),
$$

for each of $e(H)-(v(H)-1)=\ell+1$ edges $e \in E(H) \backslash E(T)$. By independence of $b_{e}$ 's, the probability that, conditioned on $\left\{b_{e}\right\}_{e \in E(T)}$, the constraints $(2.1 .1)$ are met is $(1 / 2)^{\ell+1}$. (It is crucial that $\operatorname{Pr}\left(b_{e}=0\right)=\operatorname{Pr}\left(b_{e}=1\right)=1 / 2$.) Hence the unconditional solvability probability for the system (1.0.1) with the underlying graph $H$ is $(1 / 2)^{\ell+1}$ as well.

Note. For a cycle $C \subseteq H$, let $b_{C}=\sum_{e \in E(C)} b_{e}$. The conditions (2.1.1) are equivalent to $b_{C}$ being even for the $\ell+1$ cycles, each formed by adding to $T$ an edge in $E(H) \backslash E(T)$. Adding the equations (1.0.1) over the edges of any cycle $C \subseteq H$, we see that necessarily $b_{C}$ is even too. Thus our proof effectively shows that

$$
\operatorname{Pr}\left\{\bigcap_{C \subseteq H}\left\{b_{C} \text { is even }\right\}\right\}=\left(\frac{1}{2}\right)^{\ell(H)+1} .
$$

Using Lemma 2.1, we express $P(S(n, p)>0)$ as the coefficient by $x^{n}$ in a formal power series. To formulate the result, introduce $C_{\ell}(x)$, the exponential generating function of a 
sequence $\{C(k, k+\ell)\}_{k \geqslant 1}$, where $C(k, k+\ell)$ is the total number of connected graphs $H$ on $[k]$ with excess $e(H)-v(H)=\ell$. Of course, $C(k, k+\ell)=0$ unless $-1 \leqslant \ell \leqslant\left(\begin{array}{l}k \\ 2\end{array}\right)-k$.

\section{Lemma 2.2.}

$$
\begin{aligned}
\operatorname{Pr}\left(S_{n}>0\right) & =N(n, p)\left[x^{n}\right] \exp \left[\frac{1}{2} \sum_{\ell \geqslant-1}\left(\frac{p}{2 q}\right)^{\ell} C_{\ell}(x)\right], \\
N(n, p) & :=n ! q^{n^{2} / 2}\left(\frac{p}{q^{3 / 2}}\right)^{n} .
\end{aligned}
$$

Proof of Lemma 2.2. The proof mimicks derivation of the "coefficient-of $x^{n}$ - expression" for the largest component size distribution in [22].

Given $\boldsymbol{\alpha}=\left\{\alpha_{k, \ell}\right\}$, such that $\sum_{k, \ell} k \alpha_{k, \ell}$, let $P_{n}(\boldsymbol{\alpha})$ denote the probability that $G(n, p)$ has $\alpha_{k, \ell}$ components $H$ with $v(H)=k$ and $e(H)-v(H)=\ell$. To compute $P_{n}(\boldsymbol{\alpha})$, we observe that there are

$$
\frac{n !}{\prod_{k, \ell}(k !)^{\alpha_{k, \ell}} \alpha_{k, \ell} !}
$$

ways to partition $[n]$ into $\sum_{k, \ell} \alpha_{k, \ell}$ subsets, with $\alpha_{k, \ell}$ subsets of cardinality $k$ and "type" $\ell$. For each such partition, there are

$$
\prod_{k, \ell}[C(k, k+\ell)]^{\alpha_{k, \ell}}
$$

ways to build $\alpha_{k, \ell}$ connected graphs $H$ on the corresponding $\alpha_{k, \ell}$ subsets, with $v(H)=k$, $e(H)-v(H)=\ell$. The probability that these graphs are induced subgraphs of $G(n, p)$ is

$$
\prod_{k, \ell}\left[p^{k+\ell} q^{\left(\begin{array}{c}
k \\
2
\end{array}\right)-(k+\ell)}\right]^{\alpha_{k, \ell}}=\left(\frac{p}{q^{3 / 2}}\right)^{n} \prod_{k, \ell}\left[\left(\frac{p}{q}\right)^{\ell} q^{k^{2} / 2}\right]^{\alpha_{k, \ell}},
$$

as $\sum_{k, \ell} k \alpha_{k, \ell}$. The probability that no two vertices from two different subsets are joined by an edge in $G(n, p)$ is $q^{r}$, where $r$ is the total number of all such pairs, i. e.

$$
\begin{aligned}
r & =\sum_{k, \ell} k^{2}\left(\begin{array}{c}
\alpha_{k, \ell} \\
2
\end{array}\right)+\frac{1}{2} \sum_{\left(k_{1}, \ell_{1}\right) \neq\left(k_{2}, \ell_{2}\right)} k_{1} k_{2} \alpha_{k_{1}, \ell_{1}} \alpha_{k_{2}, \ell_{2}} \\
& =-\frac{1}{2} \sum_{k, \ell} k^{2} \alpha_{k, \ell}+\frac{1}{2}\left(\sum_{k, \ell} k \alpha_{k, \ell}\right)^{2} \\
& =-\frac{1}{2} \sum_{k, \ell} k^{2} \alpha_{k, \ell}+\frac{n^{2}}{2} .
\end{aligned}
$$

Multiplying the pieces,

$$
P_{n}(\boldsymbol{\alpha})=N(n, p) \prod_{k, \ell} \frac{1}{\alpha_{k, \ell} !}\left[\frac{(p / q)^{\ell} C(k, k+\ell)}{k !}\right]^{\alpha_{k, \ell}} .
$$


So, using Lemma 2.1,

$$
\operatorname{Pr}\left(S_{n}>0\right)=N(n, p) \sum_{\alpha} \prod_{k, \ell} \frac{1}{\alpha_{k, \ell} !}\left[\frac{(1 / 2)^{\ell+1}(p / q)^{\ell} C(k, k+\ell)}{k !}\right]^{\alpha_{k, \ell}} .
$$

Notice that dropping factors $(1 / 2)^{\ell+1}$ on the right, we get 1 instead of $\operatorname{Pr}\left(S_{n}>0\right)$ on the left, i.e.

$$
1=N(n, p) \sum_{\alpha} \prod_{k, \ell} \frac{1}{\alpha_{k, \ell} !}\left[\frac{(p / q)^{\ell} C(k, k+\ell)}{k !}\right]^{\alpha_{k, \ell}} .
$$

So, multiplying both sides of (2.1.4) by $\frac{x^{n}}{N(n, p)}$ and summing over $n \geqslant 0$,

$$
\begin{aligned}
\sum_{n} x^{n} \frac{\operatorname{Pr}\left(S_{n}>0\right)}{N(n, p)} & =\sum_{\sum_{k, \ell} k \alpha_{k, \ell}<\infty} \prod_{k, \ell} \frac{x^{k \alpha_{k, \ell}}}{\alpha_{k, \ell} !}\left[\frac{(1 / 2)^{\ell+1}(p / q)^{\ell} C(k, k+\ell)}{k !}\right]^{\alpha_{k, \ell}} \\
& =\exp \left[\frac{1}{2} \sum_{\ell}(p / 2 q)^{\ell} \sum_{k} \frac{C(k, k+\ell) x^{k}}{k !}\right] \\
& =\exp \left[\frac{1}{2} \sum_{\ell}(p / 2 q)^{\ell} C_{\ell}(x)\right] .
\end{aligned}
$$

We hasten to add that the series on the right, whence the one on the left, converges for $x=0$ only. Indeed, using (2.1.5) instead of (2.1.4),

$$
\exp \left[\sum_{\ell}(p / q)^{\ell} C_{\ell}(x)\right]=\sum_{n} x^{n} \frac{1}{N(n, p)}=\sum_{n} \frac{\left(\frac{x q^{3 / 2}}{p}\right)^{n}}{n ! q^{n^{2} / 2}}=\infty,
$$

for each $x>0$. Therefore, setting $p / 2 q=p_{1} / q_{1},\left(q_{1}=1-p_{1}\right)$,

$$
\sum_{\ell}(p / 2 q)^{\ell} C_{\ell}(x)=\sum_{\ell}\left(p_{1} / q_{1}\right)^{\ell} C_{\ell}(x)=\infty, \quad \forall x>0,
$$

as well.

Note. Setting $p / q=w, x=y w$, in (2.1.7), so that $p=w /(w+1), q=1 /(w+1)$, we obtain a well known (exponential) identity, e. g. Janson et al [13],

$$
\exp \left[\sum_{\ell \geqslant-1} w^{\ell} C_{\ell}(y w)\right]=\sum_{n \geqslant 0} \frac{y^{n}}{n !}(w+1)^{\left(\begin{array}{c}
n \\
2
\end{array}\right)} ;
$$

the right expression (the left exponent resp.) is a bivariate generating function for graphs (connected graphs resp.) $G$ enumerated by $v(G)$ and $e(G)$. Here is a similar identity involving generating functions of connected graphs $G$ with a fixed positive excess,

$$
\exp \left[\sum_{\ell \geqslant 1} w^{\ell} C_{\ell}(x)\right]=\sum_{r \geqslant 0} w^{r} E_{r}(x),
$$


where $E_{0}(x) \equiv 1$, and, for $\ell \geqslant 1, E_{\ell}(x)$ is the exponential generating function of graphs $G$ without tree components and unicyclic components, that have excess $\ell(G)=e(G)-$ $v(G)=\ell$, see [13]. In the light of Lemma (2.2), we will need an expansion

$$
\exp \left[\frac{1}{2} \sum_{\ell \geqslant 1} w^{\ell} C_{\ell}(x)\right]=\sum_{r \geqslant 0} w^{r} F_{r}(x)
$$

Like $E_{r}(x)$, each power series $F_{r}(x)$ has nonnegative coefficients, and converges for $|x|<$ $e^{-1}$.

By Lemma 2.2 and (2.1.8),

$$
\begin{aligned}
\operatorname{Pr}\left(S_{n}>0\right) & =N(n, p) \sum_{r \geqslant 0}\left(\frac{p}{2 q}\right)^{r}\left[x^{n}\right]\left\{e^{H(x)} F_{r}(x)\right\} ; \\
H(x) & :=\frac{q}{p} C_{-1}(x)+\frac{1}{2} C_{0}(x) .
\end{aligned}
$$

Interchange of $\left[x^{n}\right]$ and the summation is justifiable as each of the functions on the right has a power series expansion with only nonnegative coefficients. That is, divergence of $\sum_{\ell}(p / 2 q)^{\ell} C_{\ell}(x)$ in (2.1.6) does not impede evaluation of $\operatorname{Pr}\left(S_{n}>0\right)$. Indirectly though this divergence does make it difficult, if possible at all, to obtain a sufficiently sharp estimate of the terms in the above sum for $r$ going to $\infty$ with $n$, needed to derive an asymptotic formula for that probability. Thus we need to truncate, one way or another, the divergent series on the right in (2.1.6). One of the properties of $C_{\ell}(x)$ discovered by Wright [25] is that each of these series converges (diverges) for $|x|<e^{-1}$ (for $|x|>e^{-1}$ resp.). So, picking $L \geqslant 0$, and restricting summation range to $\ell \in[-1, L]$, we definitely get a series convergent for $|x|<e^{-1}$. What is then a counterpart of $\operatorname{Pr}\left(S_{n}>0\right)$ ? Perusing the proof of Lemma 2.2, we easily see the answer.

Let $G$ be a graph with components $H_{1}, H_{2}, \ldots$ Define $\mathcal{E}(G)$, a maximum excess of $G$, by

$$
\mathcal{E}(G)=\max _{i}\left[e\left(H_{i}\right)-v\left(H_{i}\right)\right]
$$

Clearly, $\mathcal{E}(G)$ is monotone increasing, i.e. $\mathcal{E}\left(G^{\prime}\right) \leqslant \mathcal{E}\left(G^{\prime \prime}\right)$ if $G^{\prime} \subseteq G^{\prime \prime}$. Let $\mathcal{E}_{n}=\mathcal{E}(G(n, p))$.

\section{Lemma 2.3.}

$$
\operatorname{Pr}\left(S_{n}>0, \mathcal{E}_{n} \leqslant L\right)=N(n, p)\left[x^{n}\right] \exp \left[\frac{1}{2} \sum_{\ell=-1}^{L}\left(\frac{p}{2 q}\right)^{\ell} C_{\ell}(x)\right],
$$

The proof of (2.1.11) is an obvious modification of that for (2.1.2).

If, using (2.1.11), we are able to estimate $\operatorname{Pr}\left(S_{n}>0, \mathcal{E}_{n} \leqslant L\right)$, then evidently we will get a lower bound of $\operatorname{Pr}\left(S_{n}>0\right)$, via

$$
\operatorname{Pr}\left(S_{n}>0\right) \geqslant \operatorname{Pr}\left(S_{n}>0, \mathcal{E}_{n} \leqslant L\right) .
$$

Crucially, the events $\left\{S_{n}>0\right\}$ and $\left\{\mathcal{E}_{n} \leqslant L\right\}$ are positively correlated. 
Lemma 2.4.

$$
\operatorname{Pr}\left(S_{n}>0\right) \leqslant \frac{\operatorname{Pr}\left(S_{n}>0, \mathcal{E}_{n} \leqslant L\right)}{\operatorname{Pr}\left(\mathcal{E}_{n} \leqslant L\right)}
$$

Note. The upshot of (2.1.12)-(2.1.13) is that

$$
\operatorname{Pr}\left(S_{n}>0\right) \sim \operatorname{Pr}\left(S_{n}>0, \mathcal{E}_{n} \leqslant L\right),
$$

provided that $L=L(n)$ is just large enough to guarantee that $\operatorname{Pr}\left(\mathcal{E}_{n} \leqslant L\right) \rightarrow 1$.

Proof of Lemma 2.4. By Lemma 2.1,

$$
\operatorname{Pr}\left(S_{n}>0, \mathcal{E}_{n} \leqslant L\right)=\mathbf{E}\left[\left(\frac{1}{2}\right)^{X(G(n, p))} \mathbf{1}_{\{\mathcal{E}(G(n, p)) \leqslant L\}}\right],
$$

where $X(G)=e(G)-n+c(G)$. Notice that $(1 / 2)^{X(G)}$ is monotone decreasing. Indeed, if a graph $G_{2}$ is obtained by adding one edge to a graph $G_{1}$, then

$$
e\left(G_{2}\right)=e\left(G_{1}\right)+1, \quad c\left(G_{2}\right) \in\left\{c\left(G_{1}\right)-1, c\left(G_{1}\right)\right\}
$$

so that $X\left(G_{2}\right) \geqslant X\left(G_{1}\right)$. Hence, using induction on $e\left(G_{2}\right)-e\left(G_{1}\right)$,

$$
G_{1} \subseteq G_{2} \Longrightarrow X\left(G_{2}\right) \geqslant X\left(G_{1}\right) \text {. }
$$

Furthermore $\mathbf{1}_{\{\mathcal{E}(G) \leqslant L\}}$ is also monotone decreasing. (For $e \notin E(G)$, if $e$ joins two vertices from the same component of $G$ then $\mathcal{E}(G+e) \geqslant \mathcal{E}(G)$ obviously. If $e$ joins two components, $H_{1}$ and $H_{2}$ of $G$, then the resulting component has an excess more than or equal to $\max \left\{\mathcal{E}\left(H_{1}\right), \mathcal{E}\left(H_{2}\right)\right\}$, with equality when one of two components is a tree.)

Now notice that each $G$ on $[n]$ is essentially a $\left(\begin{array}{l}n \\ 2\end{array}\right)$-long tuple $\boldsymbol{\delta}$ of $\{0,1\}$-valued variables $\delta_{(i, j)}, \delta_{(i, j)}=1$ meaning that $(i, j) \in E(G)$. So, a graph function $f(G)$ can be unambigiously written as $f(\boldsymbol{\delta})$. Importantly, a monotone decreasing (increasing) graph function is a monotone decreasing (increasing) function of the code $\boldsymbol{\delta}$. For the random graph $G(n, p)$, the components of $\boldsymbol{\delta}$ are independent random variables. According to an FKG-type inequality, see Grimmett and Stirzaker [12] for instance, for any two decreasing (two increasing) functions $f(\boldsymbol{Y}), g(\boldsymbol{Y})$ of a vector $\boldsymbol{Y}$ with independent components,

$$
\mathbf{E}[f(\boldsymbol{Y}) g(\boldsymbol{Y})] \geqslant \mathbf{E}[f(\boldsymbol{Y})] \mathbf{E}[g(\boldsymbol{Y})]
$$

Applying this inequality to $(1 / 2)^{X(\boldsymbol{\delta})} \mathbf{1}_{\{\mathcal{E}(\boldsymbol{\delta}) \leqslant L\}}$, we obtain

$$
\begin{aligned}
\operatorname{Pr}\left(S_{n}>0, \mathcal{E}_{n} \leqslant L\right) & \geqslant \mathbf{E}\left[\left(\frac{1}{2}\right)^{X(G(n, p))}\right] \mathbf{E}\left[\mathbf{1}_{\{\mathcal{E}(G(n, p)) \leqslant L\}}\right] \\
& =\operatorname{Pr}\left(S_{n}>0\right) \operatorname{Pr}\left(\mathcal{E}_{n} \leqslant L\right) .
\end{aligned}
$$


Thus our next step is to determine how large $\mathcal{E}(G(n, p))$ is typically, if

$$
p=\frac{1+\lambda n^{-1 / 3}}{n}, \quad \lambda=o\left(n^{1 / 3}\right) .
$$

For $p=c / n, c<1$, it was shown in Pittel [21] that

$$
\text { lim } \operatorname{Pr}(G(n, p) \text { does not have a cycle })=(1-c)^{1 / 2} \exp \left(c / 2+c^{2} / 4\right) .
$$

From this result and monotonicity of $\mathcal{E}(G)$, it follows that, for $p$ in (2.1.14),

$$
\lim \operatorname{Pr}(\mathcal{E}(G(n, p)) \geqslant 0)=1 .
$$

If $\lambda \rightarrow-\infty$, then we also have

$$
\lim \operatorname{Pr}(\mathcal{E}(G(n, p))>0)=0,
$$

that is $\mathcal{E}(G(n, p)) \leqslant 0$ with high probability (whp). (The proof of (2.1.15) mimicks Łuczak's proof [15] of an analogous property of $G(n, m)$, with $n^{-2 / 3}(n / 2-m) \rightarrow \infty$.)

Furthermore, by Theorem 1 in [17], and monotonicity of $\mathcal{E}(G(n, p))$, it follows that $\mathcal{E}(G(n, p))$ is bounded in probability (is $O_{P}(1)$, in short), if $\lim \sup \lambda<\infty$.

Finally, suppose that $\lambda \rightarrow \infty$. Let $\mathcal{L}(G(n, m))$ denote the total excess of the number of edges over the number of vertices in the complex components of $G(n, m)$, i. e. the components that are neither trees nor unicyclic. According to a limit theorem for $\mathcal{L}\left(G\left(n, m=(n / 2)\left(1+\lambda n^{-1 / 3}\right)\right)\right)$ from [13], $\mathcal{L}(G(n, m)) / \lambda^{3} \rightarrow 2 / 3$, in probability. According to Euczak [15], whp $G(n, m)$ has exactly one complex component. So whp $\mathcal{E}(G(n, m))=\mathcal{L}(G(n, m))$, i. e. $\mathcal{E}(G(n, m)) / \lambda^{3} \rightarrow 2 / 3$ in probability, as well.

Now, if

$$
m^{\prime}=N p+O(\sqrt{N p q}), \quad N:=\left(\begin{array}{l}
n \\
2
\end{array}\right)
$$

then

$$
m^{\prime}=\frac{n}{2}\left(1+\lambda^{\prime} n^{-1 / 3}\right), \quad \lambda^{\prime}:=\lambda\left(1+O\left(n^{-1 / 6}\right)\right) .
$$

Therefore, in probability,

$$
\frac{\mathcal{E}\left(G\left(n, m^{\prime}\right)\right)}{\lambda^{3}} \rightarrow \frac{2}{3},
$$

as well. From a general "transfer principle" ( [5], [16]) it follows then that

$$
\frac{\mathcal{E}(G(n, p))}{\lambda^{3}} \rightarrow \frac{2}{3}
$$

in probability, too.

This discussion justifies the following choice of $L$ :

$$
L= \begin{cases}0, & \text { if } \lim \lambda=-\infty, \\ u \rightarrow \infty \text { however slowly, }, & \text { if } \lambda=O(1), \\ \lambda^{3}, & \text { if } \lambda \rightarrow \infty, \lambda=o\left(n^{1 / 12}\right) .\end{cases}
$$




\subsection{Generating functions}

First, some basic facts about the generating functions $C_{\ell}(x)$ and $E_{\ell}(x)$. Introduce a tree function $T(x)$, the exponential generating function of $\left\{k^{k-1}\right\}$, the counts of rooted trees on $[k], k \geqslant 1$. It is well known that the series

$$
T(x)=\sum_{k \geqslant 1} \frac{x^{k}}{k !} k^{k-1}
$$

has convergence radius $e^{-1}$, and that

$$
T(x)=x e^{T(x)}, \quad|x| \leqslant e^{-1}
$$

in particular, $T\left(e^{-1}\right)=1$. (This last fact has a probabilistic explanation: $\left\{\frac{k^{k-1}}{e^{k} k !}\right\}$ is the distribution of a total progeny in a branching process with an immediate family size being Poisson (1) distributed.) $T(x)$ is a building block for all $C_{\ell}(x)$. Namely, (Moon [20], Wright [25], Bagaev [1] resp.),

$$
\begin{aligned}
C_{-1}(x) & =T(x)-\frac{1}{2} T^{2}(x), \\
C_{0}(x) & =\frac{1}{2}\left[\ln \frac{1}{1-T(x)}-T(x)-\frac{1}{2} T^{2}(x)\right], \\
C_{1}(x) & =\frac{T^{4}(x)(6-T(x))}{24(1-T(x))^{3}},
\end{aligned}
$$

and ultimately, for all $\ell>0$,

$$
C_{\ell}(x)=\sum_{d=0}^{3 \ell+2} \frac{c_{\ell, d}}{(1-T(x))^{3 \ell-d}}
$$

$c_{\ell, d}$ being constants, Wright [25]. Needless to say, $|x|<e^{-1}$ in all the formulas. One should rightfully anticipate though that the behaviour of $C_{\ell}(x)$ for $x$ 's close to $e^{-1}$ is going to determine an asymptotic behaviour of $\operatorname{Pr}\left(S_{n}>0, \mathcal{E}_{n} \leqslant L\right)$. And so the $(d=0)$-term in (2.2.3) might well be the only term we would need eventually. In this context, it is remarkable that in a follow-up paper [26] Wright was able to show that, for $c_{\ell}:=c_{\ell, 0}>0$, $d_{\ell}:=-c_{\ell, 1}>0,(\ell \geqslant 1)$,

$$
\frac{c_{\ell}}{(1-T(x))^{3 \ell}}-\frac{d_{\ell}}{(1-T(x))^{3 \ell-1}} \leqslant_{c} C_{\ell}(x) \leqslant c \frac{c_{\ell}}{(1-T(x))^{3 \ell}} .
$$

(We write $\sum_{j} a_{j} x^{j} \leqslant \sum_{c} b_{j} x^{j}$ when $a_{j} \leqslant b_{j}$ for all $j$. ) In the same paper he also demonstrated existence of a constant $c>0$ such that

$$
c_{\ell} \sim c\left(\frac{3}{2}\right)^{\ell}(\ell-1) !, \quad d_{\ell} \sim c\left(\frac{3}{2}\right)^{\ell} \ell !, \quad(\ell \rightarrow \infty) .
$$


Later Bagaev and Dmitriev [2] showed that $c=(2 \pi)^{-1}$. By now there have been found other proofs of this fact. See, for instance, Bender et al [3] for an asymptotic expansion of $c_{\ell}$ due to Meerteens, and Euczak et al [17] for a rather elementary proof based on the behavior of the component size distribution for the critical $G(n, m)$.

Turn to $E_{r}(x), r \geqslant 1$. It was shown in [13] that, analogously to (2.2.3),

$$
\begin{aligned}
E_{r}(x) & =\sum_{d=0}^{5 r} \frac{\varepsilon_{r, d}}{(1-T(x))^{3 r-d}}, \\
\varepsilon_{r, d} & =\frac{(6 r-2 d) ! Q_{d}(r)}{2^{5 r} 3^{2 r-d}(3 r-d) !(2 r-d) !},
\end{aligned}
$$

where $Q_{0}(r)=1$, and, for $d>0, Q_{d}(r)$ is a polynomial of degree $d$. By Stirling's formula,

$$
\varepsilon_{r}:=\varepsilon_{r, 0} \sim(2 \pi)^{-1 / 2}\left(\frac{3}{2}\right)^{r} r^{r-1 / 2} e^{-r}, \quad r \rightarrow \infty .
$$

Formally differentiating both sides of (2.1.8) with respect to $w$ and equating coefficients by $w^{\ell-1}$, we get a recurrence relation

$$
r E_{r}(x)=\sum_{k=1}^{r} k C_{k}(x) E_{r-k}(x) .
$$

By (2.2.3) and (2.2.6), the highest power of $(1-T(x))^{-1}$ on both sides of $(2.2 .8)$ is $3 r$, and equating the two coefficients we get a recurrence relation involving $\varepsilon_{r}$ and $c_{r}$,

$$
r \varepsilon_{r}=\sum_{k=1}^{r} k c_{k} \varepsilon_{r-k}, \quad r \geqslant 1
$$

With these preliminaries out of the way, we turn to the formula (2.1.11) for $\operatorname{Pr}\left(S_{n}>\right.$ $\left.0, \mathcal{E}_{n} \leqslant L\right)$. Notice upfront that, for $L=0$-arising when $\lambda \rightarrow-\infty$-we simply have

$$
\operatorname{Pr}\left(S_{n}>0, \mathcal{E}_{n} \leqslant 0\right)=N(n, p)\left[x^{n}\right] e^{H(x)}, \quad H(x)=\frac{q}{p} C_{-1}(x)+\frac{1}{2} C_{0}(x) .
$$

The next Lemma provides a counterpart of (2.1.10) and (2.2.10) for $L \in[1, \infty)$.

Lemma 2.5. Given $L \in[1, \infty)$,

$$
\operatorname{Pr}\left(S_{n}>0, \mathcal{E}_{n} \leqslant L\right)=N(n, p) \sum_{r=0}^{\infty}\left(\frac{p}{2 q}\right)^{r}\left[x^{n}\right]\left\{e^{H(x)} F_{r}^{L}(x)\right\},
$$

where $\left\{F_{r}^{L}(x)\right\}$ is determined by a recurrence relation

$$
r F_{r}^{L}(x)=\frac{1}{2} \sum_{k=1}^{r \wedge L} k C_{k}(x) F_{r-k}^{L}(x), \quad r \geqslant 1,
$$

and $F_{0}^{L}(x)=1 .($ Here $a \wedge b:=\min \{a, b\}$. 
Proof of Lemma 2.5. Clearly

$$
\exp \left(\frac{1}{2} \sum_{\ell=1}^{L} w^{\ell} C_{\ell}(x)\right)=\sum_{r=0}^{\infty} w^{r} F_{r}^{L}(x)
$$

where $F_{r}^{L}(x)$ are some power series, with nonnegative coefficients, convergent for $|x|<e^{-1}$. This identity implies that

$$
\exp \left(\sum_{\ell=1}^{L} w^{\ell} C_{\ell}(x)\right)=\left(\sum_{r=0}^{\infty} w^{r} F_{r}^{L}(x)\right)^{2}
$$

Differentiating this with respect to $w$ and replacing $\exp \left(\sum_{\ell=1}^{L} w^{\ell} C_{\ell}(x)\right)$ on the left of the resulting identity with $\left(\sum_{s=0}^{\infty} w^{s} F_{s}^{L}(x)\right)^{2}$, we get, after multiplying by $w$,

$$
\left(\sum_{s=0}^{\infty} w^{s} F_{s}^{L}(x)\right)\left(\sum_{\ell=1}^{L} \ell w^{\ell} C_{\ell}(x)\right)=2 \sum_{r=1}^{\infty} r w^{r} F_{r}^{L}(x) .
$$

Equating the coefficients by $w^{r}, r \geqslant 1$, of the two sides we obtain the recurrence (2.2.12).

The recurrence (2.2.12) yields a very useful information about $F_{r}^{L}(x)$.

Lemma 2.6. Let $L>0$. For $r \geqslant 0$,

$$
F_{r}^{L}(x)=\sum_{d=0}^{5 r} \frac{f_{r, d}^{L}}{(1-T(x))^{3 r-d}}
$$

and, denoting $f_{r}^{L}=f_{r, 0}^{L}, g_{r}^{L}=-f_{r, 1}^{L}$

$$
\frac{f_{r}^{L}}{(1-T(x))^{3 r}}-\frac{g_{r}^{L}}{(1-T(x))^{3 r-1}} \leqslant{ }_{c} F_{r}^{L}(x) \leqslant c \frac{f_{r}^{L}}{(1-T(x))^{3 r}} .
$$

Furthermore the leading coefficients $f_{r}^{L}, g_{r}^{L}$ satisfy a recurrence relation

$$
\begin{aligned}
& r f_{r}^{L}=\frac{1}{2} \sum_{k=1}^{r \wedge L} k c_{k} f_{r-k}^{L} ; \quad f_{0}^{L}=1 \\
& r g_{r}^{L}=\frac{1}{2} \sum_{k=1}^{r \wedge L} k c_{k} g_{r-k}^{L}+\frac{1}{2} \sum_{k=1}^{r \wedge L} k d_{k} f_{r-k}^{L} ; \quad g_{0}^{L}=0
\end{aligned}
$$

so, in particular, $f_{r}^{L}>0$ and $g_{r}^{L}>0$ for $r>0$. 
Note. 1. This Lemma and its proof are similar to those for the generating functions $E_{r}(x)$ obtained in [10].

Proof of Lemma 2.6. (a) We prove (2.2.14) by induction on $r$. (2.2.14) holds for $r=0$ as $F_{0}^{L}(x) \equiv 1$ and $f_{0,0}^{L}=f_{0}^{L}=1$. Further, by (2.2.12) and (2.2.3),

$$
F_{1}^{L}(x)=\frac{1}{2} C_{1}(x)=\frac{1}{2} \sum_{d=0}^{5} \frac{c_{1, d}}{(1-T(x))^{3-d}},
$$

i. e. (2.2.14) holds for $r=1$ too. Assume that $r \geqslant 2$ and that (2.2.14) holds for for $r^{\prime} \in[1, r-1]$. Then, by (2.2.12), (2.2.3) and inductive assumption,

$$
\begin{aligned}
F_{r}^{L}(x) & =\frac{1}{2 r} \sum_{k=1}^{r \wedge L} k C_{k}(x) F_{r-k}^{L}(x) \\
& =\frac{1}{2 r} \sum_{k=1}^{r \wedge L} k \sum_{d=0}^{3 k+2} \frac{c_{k, d}}{(1-T(x))^{3 k-d}} \sum_{d_{1}=0}^{5(r-k)} \frac{f_{r-k, d_{1}}^{L}}{(1-T(x))^{3(r-k)-d_{1}}} \\
& =\frac{1}{2 r} \sum_{k=1}^{r \wedge L} k \sum_{d \leqslant 3 k+2, d_{1} \leqslant 5(r-k)} \frac{c_{k, d} f_{r-k, d_{1}}^{L}}{(1-T(x))^{3 r-\left(d+d_{1}\right)}} .
\end{aligned}
$$

Here

$$
0 \leqslant d+d_{1} \leqslant 3 k+2+5(r-k)=5 r-2(k-1) \leqslant 5 r,
$$

so (2.2.14) holds for $r$ as well.

(b) Plugging (2.2.14) and (2.2.3) into (2.2.12) we get

$$
\sum_{d=0}^{5 r} \frac{f_{r, d}^{L}}{(1-T(x))^{3 r-d}}=\sum_{k=1}^{r \wedge L} \frac{k}{2 r} \sum_{d_{1}=0}^{3 k+2} \frac{c_{k, d_{1}}}{(1-T(x))^{3 k-d_{1}}} \sum_{d_{2}=0}^{5(r-k)} \frac{f_{r-k, d_{2}}^{L}}{(1-T(x))^{3(r-k)-d_{2}}} .
$$

Equating the coefficients by $(1-T(x))^{-3 r}$ (by $(1-T(x))^{-3 r+1}$ resp.) on the right and on the left, we obtain (2.2.16) ((2.2.17) resp.).

(c) For $r=0,(2.2 .15)$ holds trivially. For $r \geqslant 1$, inductively we have: by (2.2.4) (upper bound) and (2.2.12), (2.2.16),

$$
\begin{aligned}
& F_{r}^{L}(x) \leqslant c \\
& \frac{1}{2 r} \sum_{k=1}^{r \wedge L} k \frac{c_{k}}{(1-T(x))^{3 k}} \frac{f_{r-k}^{L}}{(1-T(x))^{3(r-k)}} \\
&=\frac{1}{(1-T(x))^{3 r}} \frac{1}{2 r} \sum_{k=1}^{r \wedge L} k c_{k} f_{r-k}^{L} \\
&=\frac{f_{r}^{L}}{(1-T(x))^{3 r}} ;
\end{aligned}
$$


furthermore, by (2.2.4) (lower bound), (2.2.12) and (2.2.16)-(2.2.17),

$$
\begin{aligned}
F_{r}^{L}(x) \geqslant & \frac{1}{2 r} \sum_{k=1}^{r \wedge L} k\left[\frac{c_{k}}{(1-T(x))^{3 k}}-\frac{d_{k}}{(1-T(x))^{3 k-1}}\right] F_{r-k}^{L}(x) \\
\geqslant_{c} & \frac{1}{2 r} \sum_{k=1}^{r \wedge L} k \frac{c_{k}}{(1-T(x))^{3 k}}\left[\frac{f_{r-k}^{L}}{(1-T(x))^{3(r-k)}}-\frac{g_{r-k}^{L}}{(1-T(x))^{3(r-k)-1}}\right] \\
& -\frac{1}{2 r} \sum_{k=1}^{r \wedge L} k \frac{d_{k}}{(1-T(x))^{3 k-1}} \cdot \frac{f_{r-k}^{L}}{(1-T(x))^{3(r-k)}} \\
= & \frac{f_{r}^{L}}{(1-T(x))^{3 r}}-\frac{1}{(1-T(x))^{3 r-1}}\left[\frac{1}{2 r} \sum_{k=1}^{r \wedge L} k c_{k} g_{r-k}^{L}+\frac{1}{2 r} \sum_{k=1}^{r \wedge L} k d_{k} f_{r-k}^{L}\right] \\
= & \frac{f_{r}^{l}}{(1-T(x))^{3 r}}-\frac{g_{r}^{L}}{(1-T(x))^{3 r-1}} .
\end{aligned}
$$

To make the bound (2.2.15) work we need to have a close look at the sequence $\left\{f_{r}^{L}, g_{r}^{L}\right\}_{r \geqslant 0}$. First of all, it follows from (2.2.16) that

$$
f_{r}^{L} \leqslant f_{r}:=f_{r}^{\infty}, \quad g_{r}^{L} \leqslant g_{r}:=g_{r}^{\infty} .
$$

That is $f_{r}$ and $-g_{r}$ are the coefficients by $(1-T(x))^{-3 r}$ and $(1-T(x))^{-3 r+1}$ in the expansion (2.2.13) for $F_{r}(x):=F_{r}^{\infty}(x)$. Now, using (2.2.13) for $L=\infty$ and (2.1.8), we see that

$$
\left(\sum_{r \geqslant 0} w^{r} F_{r}(x)\right)^{2}=\sum_{r \geqslant 0} w^{r} E_{r}(x) .
$$

So, equating the coefficients by $w^{r}, r \geqslant 0$, we get

$$
\sum_{k=0}^{r} F_{k}(x) F_{r-k}(x)=E_{r}(x) .
$$

Plugging (2.2.6) and (2.2.14) (with $L=\infty$ ), and comparing coefficients by $(1-T(x))^{-3 r}$ $\left((1-T(x))^{-3 r+1}\right.$, resp.), we obtain

$$
\sum_{k=0}^{r} f_{k} f_{r-k}=\varepsilon_{r, 0} ; \quad 2 \sum_{k=0}^{r} f_{k} g_{r-k}=-\varepsilon_{r, 1} .
$$

In particular,

$$
f_{r} \leqslant \frac{1}{2} \varepsilon_{r, 0}, \quad g_{r} \leqslant-\frac{1}{2} \varepsilon_{r, 1} .
$$


Consequently, using (2.2.6) for $r \geqslant 2$ and $d=0$,

$$
\begin{aligned}
f_{r} & =\frac{1}{2} \varepsilon_{r, 0}-\frac{1}{2} \sum_{k=1}^{r-1} f_{k} f_{r-k} \geqslant \frac{1}{2} \varepsilon_{r, 0}-\frac{1}{2} \sum_{k=1}^{r-1} \frac{1}{2} \varepsilon_{k, 0} \frac{1}{2} \varepsilon_{r-k, 0} \\
& \geqslant \frac{\varepsilon_{r, 0}}{2}\left(1-\frac{1}{4} \sum_{j=1}^{r-1}\left(\begin{array}{l}
r \\
j
\end{array}\right)^{-1} \frac{\left(\begin{array}{l}
r \\
j
\end{array}\right)\left(\begin{array}{l}
2 r \\
2 j
\end{array}\right)\left(\begin{array}{l}
3 r \\
3 j
\end{array}\right)}{\left(\begin{array}{l}
6 r \\
6 j
\end{array}\right)}\right) \\
& \geqslant \frac{\varepsilon_{r, 0}}{2}\left(1-\frac{1}{4} \sum_{j=1}^{r-1}\left(\begin{array}{l}
r \\
j
\end{array}\right)^{-1}\right) \\
& \geqslant \frac{\varepsilon_{r, 0}}{2}(1-1 / r),
\end{aligned}
$$

that is

$$
\frac{\varepsilon_{r, 0}}{2}(1-1 / r) \leqslant f_{r} \leqslant \frac{\varepsilon_{r, 0}}{2} \sim \frac{1}{2 \sqrt{2 \pi}}\left(\frac{3}{2}\right)^{r} r^{r-1 / 2} e^{-r},(r \rightarrow \infty),
$$

see (2.2.7). Furthermore, using (2.2.6) for $r>0$ and $d=1$,

$$
g_{r} \leqslant_{b}\left(\frac{3}{2}\right)^{r} r^{r+1 / 2} e^{-r} .
$$

And one can prove a matching lower bound for $g_{r}$. Hence, like $\varepsilon_{r}, f_{r}, g_{r}$ grow essentially as $r^{r}$, too fast for $F_{r}(x)=F_{r}^{\infty}(x)$ to be useful for asymptotic estimates. The next Lemma (last in this subsection) shows that, in a pleasing contrast, $f_{r}^{L}, g_{r}^{L}$ grow much slower when $r \gg L$.

Lemma 2.7. There exists $L_{0}$ such that, for $L \geqslant L_{0}$,

$$
f_{r}^{L} \leqslant_{b}\left(\frac{3 L}{2 e}\right)^{r}, \quad g_{r}^{L} \leqslant_{b} r\left(\frac{3 L}{2 e}\right)^{r}, \quad \forall r \geqslant 0 .
$$

Proof of Lemma 2.7. (a) It is immediate from (2.2.18), (2.2.19) that, for some absolute constant $A$ and all $L>0$,

$$
f_{r}^{L}=f_{r} \leqslant A\left(\frac{3 L}{2 e}\right)^{r}, \quad g_{r}^{L}=g_{r} \leqslant A r\left(\frac{3 L}{2 e}\right)^{r} \quad 0 \leqslant r \leqslant L .
$$

Let us prove existence an integer $L>0$, with a property: if for some $s \geqslant L$ and all $t \leqslant s$,

$$
f_{t}^{L} \leqslant A\left(\frac{3 L}{2 e}\right)^{t}, \quad g_{t}^{L} \leqslant A t\left(\frac{3 L}{2 e}\right)^{t}
$$

then

$$
f_{s+1}^{L} \leqslant A\left(\frac{3 L}{2 e}\right)^{s+1}, \quad g_{s+1}^{L} \leqslant A(s+1)\left(\frac{3 L}{2 e}\right)^{s+1}
$$


By (2.2.16), (2.2.21), and (2.2.5), there exists an absolute constant $B>0$ such that

$$
(s+1) f_{s+1}^{L} \leqslant A B\left(\frac{3 L}{2 e}\right)^{s+1} L^{1 / 2} \sum_{k=1}^{L}\left(\frac{k}{L}\right)^{k} .
$$

A function $(x / L)^{x}$ attains its minimum on $[0, L]$ at $x=L / e$, and it is easy to show that

$$
(x / L)^{x} \leqslant \begin{cases}e^{-x}, & x \leqslant L / e, \\ e^{-(L-x)(3-e) / 2}, & x \geqslant L / e .\end{cases}
$$

Since $s+1 \geqslant L$, we obtain then

$$
\begin{aligned}
f_{s+1}^{L} & \leqslant A B\left(\frac{1}{1-e^{-1}}+\frac{1}{1-e^{-(3-e) / 2}}\right) \cdot L^{-1 / 2}\left(\frac{3 L}{2 e}\right)^{s+1} \\
& \leqslant A\left(\frac{3 L}{2 e}\right)^{s+1}
\end{aligned}
$$

if we choose

$$
L \geqslant L_{1}:=B^{2}\left(\frac{1}{1-e^{-1}}+\frac{1}{1-e^{-(3-e) / 2}}\right)^{2} .
$$

Likewise, by (2.2.17), (2.2.21) and (2.2.5),

$$
\begin{aligned}
(s+1) g_{s+1}^{L} \leqslant & A B(s+1)\left(\frac{3 L}{2 e}\right)^{s+1} L^{1 / 2} \sum_{k=1}^{L}\left(\frac{k}{L}\right)^{k} \\
& +A B^{\prime}(s+1)\left(\frac{3 L}{2 e}\right)^{s+1} L^{1 / 2} \sum_{k=1}^{L}\left(\frac{k}{L}\right)^{k}
\end{aligned}
$$

so that

$$
g_{s+1}^{L} \leqslant A(s+1)\left(\frac{3 L}{2 e}\right)^{s+1},
$$

if we choose

$$
L \geqslant L_{2}:=\left(B+B^{\prime}\right)^{2}\left(\frac{1}{1-e^{-1}}+\frac{1}{1-e^{-(3-e) / 2}}\right)^{2} .
$$

Thus, picking $L=\max \left\{L_{1}, L_{2}\right\}=L_{2}$, we can accomplish the inductive step, from $s(\geqslant L)$ to $s+1$, showing that, for this $L,(2.2 .20)$ holds for all $t$.

Combining (2.2.10), Lemma 2.5, Lemma 2.6, we bound $\operatorname{Pr}\left(S_{n}>0, \mathcal{E}_{n} \leqslant L\right)$.

Proposition 2.1. Let $L \in[0, \infty)$. Then

$$
\Sigma_{1} \leqslant \operatorname{Pr}\left(S_{n}>0, \mathcal{E}_{n} \leqslant L\right) \leqslant \Sigma_{2} .
$$


Here

$$
\begin{aligned}
& \Sigma_{1}=N(n, p) \sum_{r \geqslant 0}\left(\frac{p}{2 q}\right)^{r}\left[x^{n}\right]\left[\frac{f_{r}^{L} e^{H(x)}}{(1-T(x))^{3 r}}-\frac{g_{r}^{L} e^{H(x)}}{(1-T(x))^{3 r-1}}\right], \\
& \Sigma_{2}=N(n, p) \sum_{r \geqslant 0}\left(\frac{p}{2 q}\right)^{r}\left[x^{n}\right] \frac{f_{r}^{L} e^{H(x)}}{(1-T(x))^{3 r}}
\end{aligned}
$$

and

$$
f_{r}^{L}\left\{\begin{array} { l l } 
{ = f _ { r } , } & { r \leqslant L , } \\
{ \leqslant ( \frac { 3 L } { 2 e } ) ^ { r } , } & { r \geqslant L , }
\end{array} \quad g _ { r } ^ { L } \left\{\begin{array}{ll}
=g_{r}, & r \leqslant L, \\
\leqslant_{b} r\left(\frac{3 L}{2 e}\right)^{r}, & r \geqslant L,
\end{array}\right.\right.
$$

with $f_{r}, g_{r}$ satisfying the conditions (2.2.18)-(2.2.19).

Note. The relations (2.2.22)-(2.2.23) indeed cover the case $L=0$, since in this case $f_{0}=1, g_{0}=0$ and $f_{r}^{L}=g_{r}^{L}=0$ for $r>0$.

\subsection{Asymptotic formula for $\operatorname{Pr}\left(S_{n}>0\right)$.}

The Proposition 2.1 makes it clear that we need to find an asymptotic formula for

$$
N(n, p) \phi_{n, w}, \quad \phi_{n, w}:=\left[x^{n}\right] \frac{e^{H(x)}}{(1-T(x))^{w}}, \quad w=0,3,6 \ldots
$$

Using $N(n, p) ! q^{n^{2} / 2}\left(p q^{-3 / 2}\right)^{n}$ and Stirling's formula for $n$ !, with some work we obtain

$$
\begin{aligned}
N(n, p)=\sqrt{2 \pi n} \exp [ & -n \frac{3}{2}+n^{2 / 3} \frac{\lambda}{2}-n^{1 / 3} \frac{\lambda^{2}}{2} \\
& \left.+\frac{\lambda^{3}}{3}+\frac{5}{4}+O\left(n^{-1 / 3}\left(1+\lambda^{4}\right)\right)\right] .
\end{aligned}
$$

The big-Oh term here is $o(1)$ if $|\lambda|=o\left(n^{1 / 12}\right)$, which is the condition of Theorem 1.1.

Turn to $\phi_{n, w}$. Since the function in question is analytic for $|x|<e^{-1}$,

$$
\phi_{n, w}=\frac{1}{2 \pi i} \oint_{\Gamma} \frac{e^{H(x)}}{x^{n+1}(1-T(x))^{w}} d x
$$

where $\Gamma$ is a simple closed contour enclosing the origin and lying in the disc $|x|<e^{-1}$. By (2.1.10), (2.2.1)-(2.2.2), the function in (2.3.1) depends on $x$ only through $T(x)$, which satisfies $T(x)=x e^{T(x)}$. This suggests introducing a new variable of integration $y$, such that $y e^{-y}=x$, i. e.

$$
y=T(x)=\sum_{k \geqslant 1} \frac{x^{k}}{k !} k^{k-1}, \quad|x|<e^{-1}
$$


Picking a simple closed contour $\Gamma^{\prime}$ in the $y$-plane such that its image under $x=y e^{-y}$ is a simple closed contour $\Gamma$ within the disc $|x|<e^{-1}$, and using (2.2.1)-(2.2.2), we obtain

$$
\begin{aligned}
\phi_{n, w} & =\frac{1}{2 \pi i} \oint_{\Gamma^{\prime}} y^{-n-1} e^{n y} \exp \left(\kappa(y)-\frac{y}{4}-\frac{y^{2}}{8}\right)(1-y)^{3 / 4-w} d y, \\
\kappa(y) & :=\frac{q}{p}\left(y-\frac{y^{2}}{2}\right)
\end{aligned}
$$

$q / p \sim n$, so $y^{-n} e^{n y} e^{\kappa(y)}$ would have fully accounted for asymptotic behavior of the integral, had it not been for the factor $(1-y)^{3 / 4-w}$. Once $\Gamma^{\prime}$ is picked, it can be replaced by any circular contour $y=\rho e^{i \theta}, \theta \in(-\pi, \pi], \rho<1$. (The condition $\rho<1$ is dictated by the factor $(1-y)^{3 / 4-w}$.) And (2.3.3) becomes

$$
\begin{aligned}
\phi_{n, w} & =\frac{1}{2 \pi} I(w), \\
I(w) & :=\int_{-\pi}^{\pi} e^{h(\rho, \theta)} \exp \left(-\rho e^{i \theta} / 4-\rho^{2} e^{i 2 \theta} / 8\right)\left(1-\rho e^{i \theta}\right)^{3 / 4-w} d \theta, \\
h(\rho, \theta) & =\frac{q}{p}\left(\rho e^{i \theta}-\frac{\rho^{2} e^{i 2 \theta}}{2}\right)+n \rho e^{i \theta}-n(\ln \rho+i \theta) .
\end{aligned}
$$

We will choose $\rho<1$ in such a way that, as a function of $\theta,\left|e^{h(\rho, \theta)}\right|$ attains its maximum at $\theta=0$. Now $\left|e^{h(\rho, \theta)}\right|=e^{f(\rho, \theta)}$, with

$$
f(\rho, \theta)=\operatorname{Re} h(\rho, \theta)=\frac{q}{p} \rho \cos \theta-\frac{q}{2 p} \rho^{2} \cos 2 \theta+n \rho \cos \theta-n \ln \rho,
$$

so that

$$
f_{\theta}^{\prime}(\rho, \theta)=\frac{2 q}{p} \rho^{2} \sin \theta\left(\cos \theta-\frac{1+n p / q}{2 \rho}\right) .
$$

Then $f_{\theta}^{\prime}(\rho, \theta)>0(<0$ resp. $)$ for $\theta<0(\theta>0$ resp. $)$ if

$$
\rho<\frac{1}{2}(1+n p / q)
$$

Let us set $\rho=e^{-a n^{-1 / 3}}$, where $a=o\left(n^{1 / 3}\right)$, since we want $\rho \rightarrow 1$. Now

$$
\frac{1}{2}(1+n p / q)>1+\frac{\lambda}{2} n^{-1 / 3}, \quad \rho \leqslant 1-a n^{-1 / 3}+\frac{a^{2}}{2} n^{-2 / 3}
$$

so (2.3.5) is obviously satisfied if

$$
a+\frac{\lambda}{2} \geqslant \frac{a}{2}
$$

(2.3.6) is trivially met if $\lambda \geqslant 0$. For $\lambda<0,|\lambda|=o\left(n^{1 / 3}\right),(2.3 .6)$ is met if $a \geqslant|\lambda|$. In all cases we will assume that $\liminf a>0$.

Why do we want $a=o\left(n^{1 / 3}\right)$ ? Because, as a function of $\rho, h(\rho, 0)$ attains its minimum at $n p / q \sim 1$, if $\lambda<0$ is fixed, and in this case $n p / q<1$, and the minimum point 
is 1 if $\lambda \geqslant 0$. So our $\rho$ is a reasonable approximation of the saddle point of $|h(\rho, \theta)|$, dependent on $\lambda$, chosen from among the feasible values, i. e. those strictly below 1 . Characteristically $\rho$ is very close to 1 , the singular point of the factor $(1-y)^{3 / 4-w}$, which is especially influential for large $w$ 's. Its presence rules out a "pain-free" application of general tools such as Watson's Lemma, (see Miller [18]).

Under (2.3.6),

$$
\left|f_{\theta}^{\prime}(\rho, \theta)\right| \geqslant \frac{a}{2} n^{2 / 3}|\sin \theta|
$$

and $\operatorname{sign} f_{\theta}^{\prime}(\rho, \theta)=-\operatorname{sign} \theta$, so that

$$
\begin{aligned}
f(\rho, \theta) & \leqslant f(\rho, 0)-\frac{a}{2} n^{2 / 3} \int_{0}^{|\theta|} \sin z d z \\
& =f(\rho, 0)-a n^{2 / 3} \sin ^{2}(\theta / 2) \\
& \leqslant f(\rho, 0)-a \pi^{-2} n^{2 / 3} \theta^{2}=h(\rho, 0)-a \pi^{-2} n^{2 / 3} \theta^{2} .
\end{aligned}
$$

Let us break the integral $I(w)$ in (2.3.4) into two parts, $I_{1}(w)$ for $|\theta| \leqslant \theta_{0}$, and $I_{2}(w)$ for $|\theta| \geqslant \theta_{0}$, where

$$
\theta_{0}=\pi n^{-1 / 3} \ln n
$$

Since $f(\rho, \theta)$ is decreasing with $|\theta|$, and $\left|1-\rho e^{i \theta}\right| \geqslant 1-\rho$, it follows from (2.3.7) that

$$
\begin{aligned}
\left|I_{2}(w)\right| & \leqslant{ }_{b}\left(1-e^{-a n^{-1 / 3}}\right)^{-w} e^{f\left(\rho, \theta_{0}\right)} \\
& \leqslant\left(a_{1} n^{-1 / 3}\right)^{-w} e^{h(\rho, 0)} \exp \left(-a \ln ^{2} n\right) \\
a_{1} & :=n^{1 / 3}\left(1-e^{-a n^{-1 / 3}}\right) .
\end{aligned}
$$

Turn to $I_{1}(w)$. This time $|\theta| \leqslant \theta_{0}$. First, let us write

$$
\rho e^{i \theta}=e^{-s n^{-1 / 3}}, \quad s=a-i t, \quad t:=n^{1 / 3} \theta ;
$$

so $|s| \leqslant a+\pi \ln n$. The second (easy) exponent in the integrand of $I_{1}(w)$ is asymptotic to $-3 / 8$, or more precisely,

$$
\begin{aligned}
-\frac{1}{4} e^{-s n^{-1 / 3}}-\frac{1}{8} e^{-2 s n^{-1 / 3}} & =Q_{2}(a)+O\left(|t| n^{-1 / 3}\right), \\
Q_{2}(a) & :=-\frac{1}{4} e^{-a n^{-1 / 3}}-\frac{1}{8} e^{-2 a n^{-1 / 3}} .
\end{aligned}
$$

Determination of a usable asymptotic formula for $h(\rho, \theta)$ is more laborious. It is convenient to set $q / p e^{-\mu n^{-1 / 3}}$; thus

$$
\mu^{1 / 3} \ln \frac{n p}{q} \geqslant n^{1 / 3} \ln \left(1+\lambda n^{-1 / 3}\right) \geqslant \lambda\left(1-\lambda n^{-1 / 3} / 2\right),
$$

and

$$
\mu-\lambda=O\left(n^{-2 / 3}+n^{-1 / 3} \lambda^{2}\right) .
$$


Using the new parameters $s$ and $\mu$ we transform the formula (2.3.4) for $h(\rho, \theta)$ to

$$
h(\rho, \theta)=n\left(e^{-(\mu+s) n^{-1 / 3}}-\frac{1}{2} e^{-(\mu+2 s) n^{-1 / 3}}+e^{-s n^{-1 / 3}}+s n^{-1 / 3}\right) .
$$

Approximating the three exponents by the 4-th degree Taylor polynomials, we obtain

$$
\begin{aligned}
h(\rho, \theta)= & n\left[\frac{3}{2}-n^{-1 / 3} \frac{\mu}{2}+n^{-2 / 3} \frac{\mu^{2}}{4}-n^{-1} \frac{\mu^{3}}{12}\right]+Q_{1}(\mu, a) \\
& +\left(\frac{\mu s^{2}}{2}+\frac{s^{3}}{3}\right)+O\left(D_{1}(t)\right) ; \\
Q_{1}(\mu, a):= & n^{-1 / 3}\left[\frac{(\mu+a)^{4}}{4 !}-\frac{(\mu+2 a)^{4}}{4 ! 2}+\frac{a^{4}}{4 !}\right] ; \\
D_{1}(t):= & n^{-1 / 3}|t|(|\lambda|+a+\ln n)^{3}+n^{-2 / 3}(|\lambda|+a+\ln n)^{5} .
\end{aligned}
$$

(Explanation: the second summand in $D_{1}(t)$ is the approximation error bound for each of the Taylor polynomials; the first summand is the common bound of $\left|(\mu+a)^{4}-(\mu+s)^{4}\right|$, $\left|(\mu+2 s)^{4}-(\mu+2 a)^{4}\right|$, and $\left|s^{4}-a^{4}\right|$, times $n^{-1 / 3}$.) And we notice immediately that both $Q_{1}(\mu, a)$ and $D_{1}(t)$ are $o(1)$ if, in addition to $|\lambda|=o\left(n^{1 / 12}\right)$, we require that $a=o\left(n^{1 / 12}\right)$ as well, a condition we assume from now on. Obviously $O\left(D_{1}(t)\right)$ absorbs the remainder term $O\left(|t| n^{-1 / 3}\right)$ from (2.3.9).

Furthermore, since

$$
n^{-1 / 3} \mu=\ln \left(\frac{n p}{q}\right)^{-1 / 3} \lambda-n^{-2 / 3} \frac{\lambda^{2}}{2}+n^{-1}\left(\frac{\lambda^{3}}{3}+1\right)+O\left(n^{-4 / 3}\left(1+\lambda^{4}\right)\right),
$$

for the cubic polynomial of $n^{-1 / 3} \mu$ in (2.3.10) we have

$$
\begin{aligned}
& n\left[\frac{3}{2}-n^{-1 / 3} \frac{\mu}{2}+n^{-2 / 3} \frac{\mu^{2}}{4}-n^{-1} \frac{\mu^{3}}{12}\right] \\
= & n \frac{3}{2}-n^{2 / 3} \frac{\lambda}{2}+n^{1 / 3} \frac{\lambda^{2}}{2}-\frac{\lambda^{3}}{2}-\frac{1}{2}+O\left(n^{-1 / 3}\left(1+\lambda^{4}\right)\right) .
\end{aligned}
$$

Observe that the first three summands are those in the exponent of the formula (2.3.2) for $N(n, p)$ times (-1).) Therefore, using (2.3.2) for $N(n, p)$,

$$
\begin{aligned}
& N(n, p) \exp \left(h(\rho, \theta)-\frac{1}{4} \rho e^{i \theta}-\frac{1}{8} \rho^{2} e^{i 2 \theta}\right) \\
= & {\left[1+O\left(D_{1}(t)\right)\right] \sqrt{2 \pi n} \cdot \exp \left(-\frac{\lambda^{3}}{6}+\frac{3}{4}+Q(\mu, a)+\frac{\mu s^{2}}{2}+\frac{s^{3}}{3}\right) ; } \\
& Q(\mu, a):=Q_{1}(\mu, a)+Q_{2}(a)+O\left(n^{-1 / 3}\left(1+\lambda^{4}\right)\right),
\end{aligned}
$$

and $Q(\mu, a)=o(1)$ as $\lambda, a=o\left(n^{1 / 12}\right)$. In particular, using (2.3.8), (2.3.10) for $\theta=0$, i. e. $s=a$, we see that

$$
N(n, p)\left|I_{2}(w)\right| \leqslant b n^{1 / 2}\left(a_{1} n^{-1 / 3}\right)^{-w} e^{-a \ln ^{2} n} \exp \left(-\frac{\lambda^{3}}{6}+\frac{\mu a^{2}}{2}+\frac{a^{3}}{3}\right) .
$$


Furthermore, switching integration from $\theta$ to $t^{1 / 3} \theta$, the contribution of the remainder term $O\left(D_{1}(t)\right)$ to $N(n, p) I_{1}(w)$ is $O\left(\delta_{n, w}\right)$,

$$
\delta_{n, w}:^{-1 / 12} \frac{(a+\ln n)^{3 / 4} e^{-\lambda^{3} / 6}}{\left(a_{1} n^{-1 / 3}\right)^{w}} \int_{-\infty}^{\infty}\left|\exp \left(\frac{\mu s^{2}}{2}+\frac{s^{3}}{3}\right)\right| D_{1}(t) d t .
$$

(Explanation: $n^{-1 / 12}=n^{1 / 2} n^{-1 / 3} n^{-1 / 4}$, with $n^{-1 / 4}$ coming from $n^{-1 / 4}(a+\pi \ln n)^{3 / 4}$, an upper bound of $\left|1-\rho e^{i \theta}\right|^{3 / 4}$, for $|\theta| \leqslant \theta_{0}$.)

Now

$$
\left|\exp \left(\frac{\mu s^{2}}{2}+\frac{s^{3}}{3}\right)\right|=\exp \left[\frac{\mu a^{2}}{2}+\frac{a^{3}}{3}-\left(\frac{\mu}{2}+a\right) t^{2}\right],
$$

where, see (2.3.6),

$$
\frac{\mu}{2}+a=\frac{\lambda}{2}+a+O\left(n^{-2 / 3}+n^{-1 / 3} \lambda^{2}\right)>0,
$$

since $\liminf a>0$, and $a \geqslant|\lambda|$ if $\lambda<0$. Hence, see (2.3.10) for $D_{1}(t)$, we have $\delta_{n, w} \leqslant b$ $\Delta_{n, w}$, where

$$
\begin{aligned}
\Delta_{n, w} & :=n^{-1 / 12+w / 3} \cdot a_{1}^{-w} \exp \left(-\frac{\lambda^{3}}{6}+\frac{\mu a^{2}}{2}+\frac{a^{3}}{3}\right) \\
& \cdot(a+\ln n)^{3 / 4}\left[n^{-1 / 3} \frac{(|\lambda|+a+\ln n)^{3}}{\mu / 2+a}+n^{-2 / 3} \frac{(|\lambda|+a+\ln n)^{5}}{(\mu / 2+a)^{1 / 2}}\right] .
\end{aligned}
$$

The denominators $\mu / 2+a,(\mu / 2+a)^{1 / 2}$ come from the integrals

$$
\int_{-\infty}^{\infty}|t|^{k} \exp \left[-\left(\frac{\mu}{2}+a\right) t^{2}\right] d t=c_{k}(\mu / 2+a)^{-(k+1) / 2}, \quad(k \geqslant 0),
$$

for $k=0,1$. Clearly $\Delta_{n, w}$ absorbs the bound (2.3.12).

Thus, switching from $\theta$ to $s=a-i n^{1 / 3} \theta$, it remains to evaluate sharply

$$
\begin{aligned}
& -i(2 \pi)^{-1 / 2} n^{1 / 6} \exp \left(-\frac{\lambda^{3}}{6}+\frac{3}{4}+Q(\mu, a)\right) \\
& \cdot \int_{s_{1}}^{s_{2}} \exp \left(\frac{\mu s^{2}}{2}+\frac{s^{3}}{3}\right)\left(1-e^{-s n^{-1 / 3}}\right)^{3 / 4-w} d s
\end{aligned}
$$

here $s_{1}=a-i n^{1 / 3} \theta_{0}, s_{2}=a+i n^{1 / 3} \theta_{0}$, and the integral is over the vertical line segment connecting $s_{1}$ and $s_{2}$. Lastly we need to estimate an error coming from replacing (1$\left.e^{-s n^{-1 / 3}}\right)^{3 / 4-w}$ with a genuinely palatable $\left(s n^{-1 / 3}\right)^{3 / 4-w}$. Using

$$
\begin{aligned}
& \left|s n^{-1 / 3}\right| \geqslant\left|1-e^{-s n^{-1 / 3}}\right| \geqslant\left|1-e^{-a n^{-1 / 3}}\right|, \quad(s=a-i t), \\
& \left|x^{u}-1\right| \leqslant u|x-1|, \quad(u \geqslant 1,|x| \leqslant 1)
\end{aligned}
$$


we have: for $u \geqslant 1$

$$
\begin{aligned}
& \left|\frac{1}{\left(1-e^{-s n^{-1 / 3}}\right)^{u}}-\frac{1}{\left(s n^{-1 / 3}\right)^{u}}\right| \leqslant \frac{1}{\left|1-e^{-a n^{-1 / 3}}\right| u}\left|1-\left(\frac{1-e^{-s n^{-1 / 3}}}{s n^{-1 / 3}}\right)^{u}\right| \\
& \leqslant \frac{u}{\left|1-e^{-a n^{-1 / 3}}\right| u}\left|1-\frac{1-e^{-s n^{-1 / 3}}}{s n^{-1 / 3}}\right| \leqslant \frac{u\left|s n^{-1 / 3}\right|}{\left|1-e^{-a n^{-1 / 3}}\right| u} \leqslant \frac{u(a+|t|) n^{-1 / 3}}{\left|1-e^{-a n^{-1 / 3}}\right|^{u}} .
\end{aligned}
$$

Also, for $s$ in question,

$$
\left|1-e^{-s n^{-1 / 3}}\right| \geqslant 0.5\left|s n^{-1 / 3}\right|
$$

So

$$
\begin{aligned}
\left|\left(1-e^{-s n^{-1 / 3}}\right)^{3 / 4}-\left(s n^{-1 / 3}\right)^{3 / 4}\right| & =\left|s n^{-1 / 3}\right|^{3 / 4}\left|\left(\frac{1-e^{-s n^{-1 / 3}}}{s n^{-1 / 3}}\right)^{3 / 4}-1\right| \\
& \leqslant{ }_{b}\left|s n^{-1 / 3}\right|^{3 / 4+1} \leqslant{ }_{b} n^{-7 / 12}(a+|t|)^{7 / 4} .
\end{aligned}
$$

Combining these two estimates, we have: for $w \in\{0,3,4, \ldots\}$,

$$
\left|\left(1-e^{-s n^{-1 / 3}}\right)^{3 / 4-w}-\left(s n^{-1 / 3}\right)^{3 / 4-w}\right| \leqslant{ }_{b}(w+1) \frac{n^{-7 / 12}(a+|t|)^{7 / 4}}{\left(a_{1} n^{-1 / 3}\right)^{w}}
$$

see (2.3.8) for $a_{1}$. Consequently, replacing $\left(1-e^{-s n^{-1 / 3}}\right)^{3 / 4-w}$ in $(2.3 .14)$ with $\left(s n^{-1 / 3}\right)^{3 / 4-w}$ incurs an additive error of order

$$
(w+1) n^{-1 / 12+w / 3} \cdot a_{1}^{-w} \exp \left(-\frac{\lambda^{3}}{6}+\frac{\mu a^{2}}{2}+\frac{a^{3}}{3}\right) \cdot n^{-1 / 3} a^{5 / 4},
$$

at most; thus the error is easily $O\left((w+1) \Delta_{n, w}\right)$, see $(2.3 .13)$ for $\Delta_{n, w}$.

While these bounds will suffice for $\lambda=O(1)$, the case $\lambda \rightarrow \infty$ requires a sharper approximation of $\left(1-e^{-s n^{-1 / 3}}\right)^{3 / 4-w}$ for $w=O\left(\lambda^{3}\right)$. We write

$$
\begin{aligned}
\left(1-e^{-s n^{-1 / 3}}\right)^{3 / 4-w} & =\left(s n^{-1 / 3}\right)^{3 / 4-w} \exp \left[(3 / 4-w) \ln \frac{1-e^{-s n^{-1 / 3}}}{s n^{-1 / 3}}\right] \\
& =\left(s n^{-1 / 3}\right)^{3 / 4-w} \exp \left[Q_{3}(w, a)+O\left(D_{3}(w, t)\right)\right] \\
Q_{3}(w, a) & :=(3 / 4-w) \ln \frac{1-e^{-a n^{-1 / 3}}}{a n^{-1 / 3}} \\
D_{3}(w, t) & :=(w+1) t n^{-1 / 3}
\end{aligned}
$$

Notice that $Q_{3}(a, w) \rightarrow 0$ as $w a=O\left(\lambda^{3} n^{1 / 12}\right)=o\left(n^{1 / 3}\right)$, and $D_{3}(t, w) \rightarrow 0$ as $w \ln n=$ 
$o\left(n^{1 / 3}\right)$. The expression (2.3.14) therefore becomes

$$
\begin{aligned}
&-i(2 \pi)^{-1 / 2} n^{-1 / 12+w / 3} \exp \left(-\frac{\lambda^{3}}{6}+\frac{3}{4}+Q(\mu, w, a)\right) \\
& \cdot \int_{s_{1}}^{s_{2}} \exp \left(\frac{\mu s^{2}}{2}+\frac{s^{3}}{3}\right) s^{3 / 4-w} d s+O\left((w+1) \tilde{\Delta}_{n, w}\right) ; \\
& Q(\mu, w, a):=Q(\mu, a)+Q_{3}(w, a) ; \\
& \tilde{\Delta}_{n, w}:=n^{-5 / 12+w / 3} \cdot a^{-w} \exp \left(-\frac{\lambda^{3}}{6}+\frac{\mu a^{2}}{2}+\frac{a^{3}}{3}\right)(a+\ln n)^{3 / 4} .
\end{aligned}
$$

Finally, after this replacement we can extend the integration to $(a-i \infty, a+i \infty)$, since the attendant additive error is easily shown to be absorbed by $(w+1) \Delta_{n, w}$ for all $w$, and by $(w+1) \tilde{\Delta}_{n, w}$ if $w=O\left(\lambda^{3}\right)$.

Lemma 2.8. Suppose that $\lambda=o\left(n^{1 / 12}\right)$. Let $a \geqslant|\lambda|$ be such that $\lim a>0, a=o\left(n^{1 / 12}\right)$. Then, denoting $\mu^{1 / 3} \ln (n p / q)$,

$$
\begin{aligned}
& N(n, p)\left[x^{n}\right] \frac{e^{H(x)}}{(1-T(x))^{w}} \\
= & -i(2 \pi)^{-1 / 2} e^{3 / 8+o(1)} n^{-1 / 12+w / 3} e^{-\mu^{3} / 6} \int_{a-i \infty}^{a+i \infty} s^{3 / 4-w} \exp \left(\frac{\mu s^{2}}{2}+\frac{s^{3}}{3}\right) d s \\
& +O\left((w+1) R_{n, w}\right),
\end{aligned}
$$

with $R_{n, w} \leqslant \Delta_{n, w}$ for all $w$, and $R_{n, w}=\Delta_{n, w} \wedge \tilde{\Delta}_{n, w}$ if $w a$ and $w \ln n$ are both $o\left(n^{-1 / 3}\right)$. Furthermore, shifting the integration line to $\{s=b+i t: t \in(-\infty, \infty)\}$ does not change the value of the integral as long as $b \wedge(\mu / 2+b)$ remains positive.

Proof of Lemma 2.8. We only have to explain preservation of the integral, and why $e^{-\lambda^{3} / 6}$ can be replaced with $e^{-\mu^{3} / 6}$. Given such a $b$, pick $T>0$ and introduce two horizontal line segments, $C_{1,2}=\{s=\alpha \pm i T: \alpha \in[a, b]\}$, the top segment and the bottom segment being respectively right and left oriented. On $C_{1} \cup C_{2}$,

$$
\operatorname{Re}\left(\frac{\mu s^{2}}{2}+\frac{s^{3}}{3}\right)=\frac{\mu \alpha^{2}}{2}+\frac{\alpha^{3}}{3}-T^{2}\left(\frac{\mu}{2}+\alpha\right),
$$

and

$$
\frac{\mu}{2}+\alpha \geqslant \frac{\mu}{2}+(a \wedge b)>0
$$

Therefore

$$
\lim _{T \rightarrow \infty} \int_{C_{1} \cup C_{2}} s^{3 / 4-w} \exp \left(\frac{\mu s^{2}}{2}+\frac{s^{3}}{3}\right) d s=0 .
$$


As for $e^{-\lambda^{3} / 6} \sim e^{-\mu^{3} / 6}$, this follows from

$$
\left|\lambda^{3}-\mu^{3}\right| \leqslant_{b} \lambda^{2}\left(n^{-2 / 3}+n^{-1 / 3} \lambda^{2}\right)=\left(n^{-1 / 3} \lambda\right)^{2}+n^{-1 / 3} \lambda^{4} \rightarrow 0 .
$$

In the context of the critical random graph $G(n, m)$, the integral appearing in $(2.3 .17)$ was encountered and studied in [13]. Following [13], introduce

$$
A(y, \mu)=\frac{e^{-\mu^{3} / 6}}{2 \pi i} \int_{a-i \infty}^{a+i \infty} s^{1-y} \exp \left(\frac{\mu s^{2}}{2}+\frac{s^{3}}{3}\right) d s .
$$

We know that this integral is well defined, and does not depend on $a$, if $a>0$ and $a>-\mu / 2$. It was shown in [13] that (1)

$$
A(y, \mu)=\frac{e^{-\mu^{3} / 6}}{3^{(y+1) / 3}} \sum_{k=0}^{\infty} \frac{\left(3^{2 / 3} \mu / 2\right)^{k}}{k ! \Gamma((y+1-2 k) / 3)},
$$

(2) $A(y, \mu) \geqslant 0$ for $y>0, A(y, \mu)>0$ for $y \geqslant 2$, and (3)

$$
A(y, \mu) \sim \begin{cases}(2 \pi)^{-1 / 2}|\mu|^{1 / 2-y}, & \mu \rightarrow-\infty, \\ \frac{e^{-\mu^{3} / 6}}{2^{y / 2} \Gamma(y / 2) \mu^{1-y / 2}}, & \mu \rightarrow \infty .\end{cases}
$$

We will also need two bounds

$$
\begin{aligned}
& A(y, \mu) \leqslant_{b} e^{2|\mu|^{3} / 3} \frac{(2 / 3)^{\frac{y+1}{3}}}{\Gamma\left(\frac{y+1}{3}\right)}, \\
& A(y, \mu) \leqslant{ }_{b}(a+\mu / 2)^{-1 / 2} a^{1-y} \exp \left(-\frac{\mu^{3}}{6}+\frac{\mu a^{2}}{2}+\frac{a^{3}}{3}\right),
\end{aligned}
$$

(the second bound holding for $y \geqslant 1$ and $a+\mu / 2>0$ ), and an asymptotic formula: if $\mu \rightarrow \infty, y \rightarrow \infty$, and $y=O\left(\mu^{3}\right)$, then

$$
A(y, \mu) \sim(2 \pi)^{-1 / 2}\left(y \xi^{-2}+\mu+2 \xi\right)^{-1 / 2} \xi^{1-y} \exp \left(-\frac{\mu^{3}}{6}+\frac{\mu \xi^{2}}{2}+\frac{\xi^{3}}{3}\right)
$$

where $\xi=\xi(y, \mu)$ is a unique positive root of

$$
\mu \xi^{2}+\xi^{3}=y
$$

Also, if $y=O\left(\lambda^{3}\right)$, then

$$
A(y, \mu) \leqslant_{b} \mu^{-1 / 2} \xi^{1-y} \exp \left(-\frac{\mu^{3}}{6}+\frac{\mu \xi^{2}}{2}+\frac{\xi^{3}}{3}\right) .
$$


(See Appendix for a proof of (2.3.22) and (2.3.23)-(2.3.24).)

With $A(y, \mu)$, we write (2.3.17) more compactly:

$$
\begin{aligned}
N(n, p) & {\left[x^{n}\right] \frac{e^{H(x)}}{(1-T(x))^{w}} } \\
= & (2 \pi)^{1 / 2} e^{3 / 8+o(1)} A(1 / 4+w, \mu) n^{-1 / 12+w / 3}+O\left((w+1) \Delta_{n, w}\right) .
\end{aligned}
$$

Let us use (2.3.25) for asymptotic evaluation of $\operatorname{Pr}\left(S_{n}>0, \mathcal{E}_{n} \leqslant L\right)$ given by (2.2.10)$(2.2 .11)$.

Case $|\lambda|=O(1)$. According to (2.1.16), we can pick $L \rightarrow \infty$ as slowly as we wish. We pick $L=(\ln n)^{1 / 4}$.

As a first step, let us estimate the overall contributions, $R_{n}^{(1)}$ and $R_{n}^{(2)}$, of the remainders $O\left((w+1) R_{n, w}\right)$ to the bounds $\Sigma_{1}$ and $\Sigma_{2}$ in Proposition 2.1. In this case we choose $a=(L)^{1 / 3}$ for each $w$, and $R_{n, w}=\Delta_{n, w}$. Consider $R_{n}^{(2)}$ first. By (2.2.19) and (2.3.13), and dropping $(3 r+1)(n p / 2 q)^{r}=(3 r+1)(1 / 2+o(1))^{r}$ factor,

$$
\begin{aligned}
& R_{n}^{(2)} \leqslant{ }_{b} n^{-1 / 12} \cdot\left(n^{-1 / 3} \ln ^{15 / 4} n\right) \\
& \cdot \exp \left(-\frac{\lambda^{3}}{6}+\frac{\mu a^{2}}{2}+\frac{a^{3}}{3}\right) \cdot \sum_{r=0}^{\infty} f_{r}^{L} a_{1}^{-3 r} .
\end{aligned}
$$

Now $a_{1} \sim a$, so by $(2.2 .23)$ and (2.2.18),

$$
\sum_{r=0}^{\infty} f_{r}^{L} a_{1}^{-3 r} \leqslant b \sum_{r \leqslant L}\left(\frac{3 r}{2 e a_{1}^{3}}\right)^{r}+\sum_{r>L}\left(\frac{3 L}{2 e a_{1}^{3}}\right)^{r} \leqslant \sum_{r \geqslant 0}\left(\frac{2}{e}\right)^{r}<\infty .
$$

So (2.3.26) becomes

$$
R_{n}^{(2)} \leqslant{ }_{b} n^{-1 / 12} \cdot n^{-1 / 3} e^{\ln ^{3 / 4} n}=n^{-5 / 12+o(1)} .
$$

Further, by (2.2.22) and $g_{0}^{L}=0$,

$$
\Sigma_{2}-\Sigma_{1}=N(n, p) \sum_{r>0}\left(\frac{p}{2 q}\right)^{r}\left[x^{n}\right] \frac{g_{r}^{L} e^{H(x)}}{(1-T(x))^{3 r-1}} .
$$

Therefore

$$
\begin{aligned}
&\left|R_{n}^{(1)}\right| \leqslant_{b} R_{n}^{(2)}+n^{-1 / 12} \cdot\left(n^{-1 / 3} \ln ^{15 / 4} n\right) \\
& \cdot \exp \left(-\frac{\lambda^{3}}{6}+\frac{\mu a^{2}}{2}+\frac{a^{3}}{3}\right) \cdot \sum_{r=1}^{\infty} g_{r}^{L} a_{1}^{-3 r+1} .
\end{aligned}
$$


So, using the bounds (2.2.19) and (2.2.23) for $g_{r}^{L}$, we conclude that $\left|R_{n}^{(1)}\right| \leqslant 2 R_{n}^{(2)}$. Thus, for $L=(\ln n)^{1 / 4}$,

$$
\Sigma_{1}^{*}+O\left(n^{-1 / 3+o(1)}\right) \leqslant \frac{\operatorname{Pr}\left(S_{n}>0, \mathcal{E}_{n} \leqslant L\right)}{(2 \pi)^{1 / 2} e^{3 / 8} n^{-1 / 12}} \leqslant \Sigma_{2}^{*}+O\left(n^{-1 / 3+o(1)}\right),
$$

where

$$
\begin{aligned}
& \Sigma_{2}^{*}=\sum_{r \geqslant 0}\left(\frac{n p}{2 q}\right)^{r} f_{r}^{L} A(1 / 4+3 r, \mu), \\
& \Sigma_{1}^{*}=\Sigma_{2}^{*}-n^{-1 / 3} \sum_{r>0}\left(\frac{n p}{2 q}\right)^{r} g_{r}^{L} A(-3 / 4+3 r, \mu) .
\end{aligned}
$$

Let us have a close look at $\Sigma_{1}^{*}$ and $\Sigma_{2}^{*}$. Write

$$
\begin{aligned}
\Sigma_{2}^{*} & =\sum_{r \leqslant L}\left(\frac{n p}{2 q}\right)^{r} f_{r} A(1 / 4+3 r, \mu)+\sum_{r>L}\left(\frac{n p}{2 q}\right)^{r} f_{r}^{L} A(1 / 4+3 r, \mu) \\
& =\Sigma_{21}^{*}+\Sigma_{22}^{*} .
\end{aligned}
$$

By $f_{r}^{L} \leqslant f_{r},(2.2 .18),(2.3 .22)$, and Stirling's formula for $\Gamma(r)=(r-1)$ !,

$$
\begin{aligned}
\sum_{22}^{*} & \leqslant b e^{2|\mu|^{3} / 3} \sum_{r>L}\left(\frac{1}{2}+O\left(|\lambda| n^{-1 / 3}\right)\right)^{r}\left(\frac{3}{2}\right)^{r} r^{r-1 / 2} e^{-r}\left(\frac{2}{3}\right)^{r} \Gamma^{-1}(r) \\
& \leqslant e^{2|\mu|^{3} / 3} \sum_{r>L}\left(\frac{2}{3}\right)^{r} \leqslant b\left(\frac{2}{3}\right)^{L} .
\end{aligned}
$$

Further, since uniformly for $r \leqslant L$,

$$
\left(1+\lambda n^{-1 / 3}\right)^{r}=\exp \left(O\left(L|\lambda| n^{-1 / 3}\right)\right)=1+O\left(n^{-1 / 3}(\ln n)^{1 / 4}\right),
$$

we have

$$
\Sigma_{21}^{*}=(1+o(1)) \sum_{r \leqslant L} \frac{f_{r}}{2^{r}} A(1 / 4+3 r, \mu) .
$$

And, analogously to $\Sigma_{22}^{*}$,

$$
\sum_{r>L} \frac{f_{r}}{2^{r}} A(1 / 4+3 r, \mu) \leqslant b\left(\frac{2}{3}\right)^{L}
$$

Therefore

$$
\Sigma_{2}^{*} \sim \sum_{r \leqslant L} \frac{f_{r}}{2^{r}} A(1 / 4+3 r, \mu) \rightarrow \sum_{r \leqslant L} \frac{f_{r}}{2^{r}} A(1 / 4+3 r, \mu) .
$$

Also, by the definition of $\Sigma_{1}^{*}$ in $(2.3 .25)$, it follows that $\left|\Sigma_{1}^{*}-\Sigma_{2}^{*}\right|$ is $O\left(n^{-1 / 3}\right)$. Hence 
Proposition 2.2. For $|\lambda|=O(1)$,

$$
\frac{\operatorname{Pr}\left(S_{n}>0, \mathcal{E}_{n} \leqslant L\right)}{(2 \pi)^{1 / 2} e^{3 / 8} n^{-1 / 12}} \sim c(\mu):=\sum_{r \geqslant 0} \frac{f_{r}}{2^{r}} A(1 / 4+3 r, \mu),
$$

and $\mu\left(=\lambda+O\left(n^{-1 / 3}\right)\right)$ can be replaced with $\lambda$, as $c(x)$ is positive and continuous for all $x$.

Case $\lambda \rightarrow \infty, \lambda=o\left(n^{1 / 12}\right)$. According to (2.1.16), we select $L=\alpha \lambda^{3}, \alpha>2 / 3$. This time we use a eqrefined version of (2.3.24), with the exponential factor sneaking behind the sum operation for $r \leqslant \alpha \lambda^{3}$, which allows us to choose $a(\leqslant 2 \lambda)$ dependent on $r$ for $r \leqslant \alpha \lambda^{3}$. Also, for those $r$ and $a, r a=O\left(\lambda^{4}\right)=o\left(n^{1 / 3}\right)$ and $r \ln n=O\left(\lambda^{3} \ln n\right)=o\left(n^{1 / 3}\right)$; so $R_{n, 3 r}=\tilde{\Delta}_{n, 3 r}$ in this range. For $r>\alpha \lambda^{3}$ we select $a=\lambda$, and here $R_{n, 3 r}=\Delta_{n, 3 r}$. (So, $a=o\left(n^{1 / 12}\right)$ throughout.) By (2.2.20),

$$
\begin{aligned}
& \exp \left(-\frac{\lambda^{3}}{6}+\frac{\lambda a^{2}}{2}+\frac{a^{3}}{3}\right) \sum_{r>\alpha \lambda^{3}}(r+1)(1 / 2+o(1))^{r} f_{r}^{L} \\
& \leqslant_{b} \exp \left(\frac{2 \lambda^{3}}{3}\right) \sum_{r>\alpha \lambda^{3}}(r+1)\left(\frac{3 \alpha \lambda^{3}(1+o(1))}{4 e \lambda^{3}}\right)^{r} \\
& \leqslant_{b} \lambda^{3} \exp \left(\frac{2 \lambda^{3}}{3}+\lambda^{3} \alpha \ln \frac{3 \alpha(1+o(1))}{4 e}\right) \\
& \leqslant_{b} \lambda^{3} \exp \left(\lambda^{3} \alpha \ln \frac{3 \alpha(1+o(1))}{4}\right),
\end{aligned}
$$

and, pushing $\alpha$ down to $2 / 3$, we can make the coefficient of $\lambda^{3}$ in the exponent arbitrarily close to

$$
\frac{2}{3} \cdot \ln \frac{1}{2}=-0.46 \ldots
$$

According to (2.2.18) and (2.3.13), it remains to bound

$$
\sum_{r \leqslant \alpha \lambda^{3}} \frac{r+1}{(r+1)^{1 / 2}} \min _{a \leqslant 2 \lambda}\left\{\exp \left(-\frac{\mu^{3}}{6}+\frac{\mu a^{2}}{2}+\frac{a^{3}}{3}\right)\left(\frac{3 r}{4 e a^{3}}\right)^{r}\right\}
$$

(we have replaced $a_{1}^{1 / 3}\left(1-e^{-a n^{-1 / 3}}\right)$ with $a$, since for $r \leqslant \alpha \lambda^{3}$,

$$
a_{1}^{3 r}=a^{3 r} e^{O\left(\lambda^{4} n^{-1 / 3}\right)} \sim a^{3 r},
$$

and $\lambda^{3} / 6$ with $\mu^{3} / 6$, see (2.3.18)). So we need to find $\min _{a \leqslant 2 \lambda} \Phi(r, a)$,

$$
\Phi(r, a):=-\frac{\mu^{3}}{6}+r \ln \left(\frac{3 r}{4 e a^{3}}\right)+\frac{\mu a^{2}}{2}+\frac{a^{3}}{3} .
$$


$\Phi(r, a)$ attains its absolute minimum at $a=\xi(r)$, a unique positive root of

$$
\mu \xi+\xi^{2}=\frac{3 r}{\xi} \leqslant \frac{3 \alpha \lambda^{3}}{\xi},
$$

i. e. $\xi(r)<2 \lambda$ if $\alpha$ is sufficiently close to $2 / 3$ from above. Further $\phi(r):=\Phi(r, \xi(r))$ attains its maximum at $\bar{r}$, a root of

$$
\begin{aligned}
\phi^{\prime}(r) & =\left.\Phi_{r}(r, \xi)\right|_{\xi=\xi(r)}+\left.\xi_{r}(r) \Phi_{\xi}(r, \xi)\right|_{\xi=\xi(r)} \\
& =\left.\Phi_{r}(r, \xi)\right|_{\xi=\xi(r)}=\ln \frac{3 r}{4}-3 \ln \xi(r)=0,
\end{aligned}
$$

i. e.

$$
\bar{r}=\frac{4}{81} \mu^{3}, \quad \bar{\xi}:=\xi(\bar{r})=\frac{\mu}{3}(<2 \lambda) .
$$

Consequently

$$
\phi(\bar{r})=-\frac{4 \mu^{3}}{27}=-\frac{4 \lambda^{3}}{27}+o(1) .
$$

From (2.3.30) it follows, via implicit differentiation, that

$$
\xi_{r}(r):=\frac{d \xi(r)}{d r}=\frac{3 \xi(r)}{6 r+\xi^{3}(r)}
$$

Hence

$$
\phi^{\prime \prime}(r)=\frac{1}{r}-3 \frac{\xi_{r}(r)}{\xi(r)}=-\frac{\mu \xi^{2}(r)}{r\left(6 r+\xi^{3}(r)\right)}<0
$$

in particular

$$
\phi^{\prime \prime}(\bar{r})=-\frac{27}{4 \mu^{3}} \sim-\frac{27}{4 \lambda^{3}} .
$$

A standard application of Laplace method yields

$$
\sum_{r \leqslant \alpha \lambda^{3}}(r+1)^{1 / 2} \min _{a \leqslant \lambda}\left\{\exp \left(-\frac{\mu^{3}}{6}+\frac{\lambda a^{2}}{2}+\frac{a^{3}}{3}\right)\left(\frac{3 r}{4 e a^{3}}\right)^{r}\right\} \leqslant{ }_{b} \lambda^{3} \exp \left(-\frac{4 \lambda^{3}}{27}\right) .
$$

Therefore, consulting (2.3.16) for $\tilde{\Delta}_{n, w}$ and (2.3.13) for $\Delta_{n, w}$, we bound $R_{n}^{(2)}$, the total contribution of the remainders $(w+1) R_{n, w}$ to the sum $\Sigma_{2}$ in $(2.2 .20)$ :

$$
\begin{aligned}
R_{n}^{(2)}=\sum_{r}(r+1) R_{n, 3 r} \leqslant & n^{-1 / 12} \exp \left(-\frac{4 \lambda^{3}}{27}\right)\left(n^{-1 / 3} \lambda^{3.75}+n^{-1 / 3} \ln ^{3 / 4} n\right) \\
& +n^{-1 / 12} \lambda^{3} e^{-0.27 \lambda^{3}}\left(n^{-1 / 3} \lambda^{4}+n^{-1 / 3} \ln ^{4} n\right) \\
\leqslant & \left(\lambda^{-1 / 4}+n^{-1 / 3+o(1)}\right) n^{-1 / 12} \exp \left(-\frac{4 \lambda^{3}}{27}\right)
\end{aligned}
$$


As for $R_{n}^{(1)}$, the total contribution of the remainders $(w+1) R_{n, w}$ to $\Sigma_{1}$ in $(2.2 .20)$, it is $O\left(R_{n}^{(2)}\right)$, just like the $\lambda=O(1)$ case. So we arrive at the counterpart of (2.3.27)-(2.3.28), with

$$
\left(\lambda^{-1 / 4}+n^{-1 / 3+o(1)}\right) \exp \left(-\frac{4 \lambda^{3}}{27}\right)
$$

taking place of $n^{-1 / 3+o(1)}$. Further, again we split $\Sigma_{2}^{*}=\Sigma_{21}^{*}+\Sigma_{22}^{*}$. To bound $\Sigma_{22}^{*}$ we use the second bound in (2.3.22) for $A(1 / 4+3 r, \mu)$ with $a \equiv \lambda$, and the bound (2.2.20) for $f_{r}^{L}$. Just like $R_{n}^{(2)}$, we obtain

$$
\begin{aligned}
& \Sigma_{22}^{*} \leqslant \lambda^{1 / 2} \exp \left(-\frac{\lambda^{3}}{6}+\frac{\lambda a^{2}}{2}+\frac{a^{3}}{3}\right) \\
& \cdot \sum_{r>\alpha \lambda^{3}}(1 / 2+o(1))^{r} f_{r}^{L} \leqslant \leqslant_{b} \lambda^{1 / 2} e^{-0.46 \lambda^{3}} .
\end{aligned}
$$

To evaluate sharply $\Sigma_{21}^{*}$, we use (2.3.23) to approximate $A(y, \mu)$ for $\varepsilon \mu \leqslant y, y=O\left(\lambda^{3}\right)$, and (2.3.24) to bound $A(y, \mu)$ for $y \leqslant \varepsilon \mu, \varepsilon>0$ sufficiently small. Invoking (2.2.18) as well, we have

$$
\sum_{\varepsilon \mu \leqslant r \leqslant \alpha \lambda^{3}}\left(\frac{n p}{2 q}\right)^{r} f_{r} A(1 / 4+3 r, \mu) \sim \frac{1}{4 \pi} \sum_{\varepsilon \mu \leqslant r \leqslant \alpha \lambda^{3}} \frac{\xi^{3 / 4} e^{\phi(r)}}{\left(r\left(\left(\frac{1}{4}+3 r\right) \xi^{-2}+\mu+2 \xi\right)\right)^{1 / 2}} ;
$$

here $\phi(r):=\min _{a} \Phi(r, a)=\Phi(r, \xi)$, see (2.3.28)-(2.3.29) for $\Phi(r, a)$ and $\xi=\xi(r)$. We know that $\phi(r)$ attains its pronounced maximum at $\bar{r}=(4 / 81) \lambda^{3}$, i. e. well within $\left[\varepsilon \mu, \alpha \lambda^{3}\right]$. Using (2.3.31)-(2.3.33), by Laplace method,

$$
\begin{aligned}
\sum_{\varepsilon \mu \leqslant r \leqslant \alpha \lambda^{3}}\left(\frac{n p}{2 q}\right)^{r} f_{r} A(1 / 4+3 r, \mu) & \sim \frac{1}{4 \pi} \frac{\bar{\xi}^{3 / 4} e^{\phi(\bar{r})}}{\left(\bar{r}\left(\left(\frac{1}{4}+3 \bar{r}\right) \bar{\xi}-2+\mu+2 \bar{\xi}\right)\right)^{1 / 2}}\left(\frac{2 \pi}{-\phi^{\prime \prime}(\bar{r})}\right)^{1 / 2} \\
& \sim \frac{1}{2(2 \pi)^{1 / 2} 3^{3 / 4}} \lambda^{1 / 4} \exp \left(-\frac{4 \lambda^{3}}{27}\right) .
\end{aligned}
$$

Applying (2.3.24), it is not difficult to show that

$$
\sum_{r \leqslant \varepsilon \mu}\left(\frac{n p}{2 q}\right)^{r} f_{r} A(1 / 4+3 r, \mu) \ll \lambda^{1 / 4} \exp \left(-\frac{4 \lambda^{3}}{27}\right) .
$$

So

$$
\Sigma_{12}^{*}:=\sum_{r \leqslant \alpha \lambda^{3}}\left(\frac{n p}{2 q}\right)^{r} f_{r} A(1 / 4+3 r, \mu) \sim \frac{1}{2(2 \pi)^{1 / 2} 3^{3 / 4}} \lambda^{1 / 4} \exp \left(-\frac{4 \lambda^{3}}{27}\right),
$$

whence (see (2.3.35))

$$
\Sigma_{2}^{*} \sim \frac{1}{2(2 \pi)^{1 / 2} 3^{3 / 4}} \lambda^{1 / 4} \exp \left(-\frac{4 \lambda^{3}}{27}\right)
$$

as well. And, analogously to the $\lambda=O(1)$ case, for $\Sigma_{1}^{*}$ defined in (2.3.28),

$$
\left|\Sigma_{1}^{*}-\Sigma_{2}^{*}\right| \ll \lambda^{1 / 4} \exp \left(-\frac{4 \lambda^{3}}{27}\right) \text {. }
$$


Proposition 2.3. For $\lambda \rightarrow \infty, \lambda=o\left(n^{1 / 12}\right)$,

$$
\operatorname{Pr}\left(S_{n}>0, \mathcal{E}_{n} \leqslant L\right) \sim n^{-1 / 12} \frac{e^{3 / 8}}{2 \cdot 3^{3 / 4}} \lambda^{1 / 4} \exp \left(-\frac{4 \lambda^{3}}{27}\right) .
$$

Proof of Proposition 2.3. The probability is asymptotic to the expression in $(2.3 .36)$ times $(2 \pi)^{1 / 2} e^{3 / 8} n^{-1 / 12}$.

Lastly,

Case $\lambda \rightarrow-\infty,|\lambda|=o\left(n^{1 / 12}\right)$. According to (2.1.16), we can pick $L=0$. By Proposition 2.1 and (2.3.17) for $w=0$, and $a \geqslant|\lambda|, a=o\left(n^{1 / 12}\right)$, we have

$$
\begin{aligned}
\operatorname{Pr}\left(S_{n}>0, \mathcal{E}_{n} \leqslant 0\right) & =N(n, p)\left[x^{n}\right] e^{H(x)} \\
& =(2 \pi)^{1 / 2} n^{-1 / 12} e^{3 / 8} A(1 / 4, \mu)+O\left(\Delta_{n, 0}\right) .
\end{aligned}
$$

Notice that

$$
\left.\left(\frac{\mu a^{2}}{2}+\frac{\alpha^{3}}{3}\right)\right|_{a=|\lambda|}=\frac{\lambda^{3}}{6}+o(1),
$$

since $\lambda^{3}-\mu^{3}=o(1)$. Setting $a=\lambda$ in (2.3.13), we obtain

$$
\Delta_{n, 0} \ll n^{-1 / 12} \cdot n^{-1 / 3} \cdot n^{-1 / 3}\left(|\lambda|^{3.75}+\ln ^{3.75} n\right) .
$$

And, by (2.3.21),

$$
A(1 / 4, \mu) \sim(2 \pi)^{-1 / 2}|\mu|^{1 / 2-1 / 4} \sim(2 \pi)^{-1 / 2}|\lambda|^{1 / 4} .
$$

Proposition 2.4. Suppose $\lambda \rightarrow-\infty,|\lambda|=o\left(n^{1 / 12}\right)$. Then

$$
\operatorname{Pr}\left(S_{n}>0, \mathcal{E}_{n} \leqslant 0\right) \sim e^{3 / 8}|\lambda|^{1 / 4} .
$$

Since in each of the three cases our $L$ is such that

$$
\lim \operatorname{Pr}\left(\mathcal{E}_{n} \leqslant L\right)=1,
$$

Propositions 2.2 -2.4 combined with the relations (2.1.12) and (2.1.13), prove the part of Theorem 1.1 about $G(n, p), \hat{p}=1 / 2$.

\section{Solvability probability: $G(n, m)$ and $\hat{p}=1 / 2$}

Our task is to show that the result for the near-critical $G(n, p), p=\left(1+\lambda n^{-1 / 3}\right) / n$, $\lambda=o\left(n^{1 / 12}\right)$, implies the analogous claim for $G(n, m), m=(n / 2)\left(1+\lambda n^{-1 / 3}\right)$. Denoting $N=\left(\begin{array}{l}n \\ 2\end{array}\right)$,

$$
\left.p=\frac{m}{N}+O\left(m^{1 / 2} N^{-1}\right)=\frac{1+n^{-1 / 3} \lambda^{\prime}}{n}, \quad \lambda^{\prime}=\lambda+O\left(n^{-1 / 6}\right)\right) .
$$


Obviously $\lambda^{\prime}=o\left(n^{1 / 12}\right)$, so

$$
\operatorname{Pr}(S(G(n, p)>0) \rightarrow 0 .
$$

Since an event $\{S(G)>0\}$ is monotone (increasing) with $G$, the general " $p$-to- $m$ " results, Bollobás [5], Łuczak [16], imply that

$$
\operatorname{Pr}(S(G(n, m)>0) \rightarrow 0,
$$

too. However we want to prove a sharp formula

$$
\operatorname{Pr}\left(S(G(n, m)>0) \sim c(\lambda) n^{-1 / 12}, \quad \lambda=o\left(n^{1 / 12}\right)\right.
$$

that is, by Proposition 2.3, the probabilities in question can be as small as $\exp \left(-n^{1 / 4-o(1)}\right)$. It turns out that in our case the arguments in [5], [16] can be sharpened to yield (3.0.2).

To start, recall the classic entropy bound

$$
\begin{array}{ll}
\operatorname{Pr}(\operatorname{Bin}(N, p) \geqslant k) \leqslant \exp [N H(k / N)], & k>N p, \\
\operatorname{Pr}(\operatorname{Bin}(N, p) \leqslant k) \leqslant \exp [N H(k / N)], & k<N p,
\end{array}
$$

where

$$
H(x):=x \ln (p / x)+(1-x) \ln (q /(1-x)) .
$$

Approximating $H(x)$ by its second degree Taylor polynomial plus a remainder term, we obtain: uniformly for $p \leqslant 1 / 2$, and $\omega \leqslant a(N p)^{1 / 6}, a>0$ being fixed,

$$
\begin{aligned}
& \operatorname{Pr}(\operatorname{Bin}(N, p) \geqslant N p+\omega \sqrt{N p q}) \leqslant{ }_{b} e^{-\omega^{2} / 2}, \\
& \operatorname{Pr}(\operatorname{Bin}(N, p) \leqslant N p-\omega \sqrt{N p q}) \leqslant{ }_{b} e^{-\omega^{2} / 2},
\end{aligned}
$$

(The bounded factor implicit in $\leqslant{ }_{b}$ notation depends on $a$.) Given $m$ and $\omega \leqslant m^{1 / 6}$, introduce $p_{1}<p_{2}$ :

$$
\begin{aligned}
& N p_{1}+\omega \sqrt{N p_{1}}=m \Longrightarrow p_{1}=(4 N)^{-1}\left(\sqrt{4 m+\omega^{2}}-\omega\right)^{2}, \\
& N p_{2}-\omega \sqrt{N p_{2}}=m \Longrightarrow p_{2}=(4 N)^{-1}\left(\sqrt{4 m+\omega^{2}}+\omega\right)^{2} .
\end{aligned}
$$

Then

$$
\begin{aligned}
\frac{N p_{2}}{\omega^{6}}>\frac{N p_{1}}{\omega^{6}} & =\frac{m}{\omega^{6}}\left(\sqrt{1+\omega^{2} / 4 m}-\omega /(2 \sqrt{m})^{2}\right. \\
& \geqslant a:=(\sqrt{2}-1)^{2},
\end{aligned}
$$

as $\omega / 2 \sqrt{m} \leqslant 0.5 m^{-1 / 3} \leqslant 1$.

Now, using $e(G)$ to denote the number of edges in a graph $G, e(G(n, p))=\operatorname{Bin}(N, p)$. So, by (3.0.3)-(3.0.5),

$$
\begin{aligned}
& \operatorname{Pr}\left(e\left(G\left(n, p_{1}\right)\right)>m\right) \leqslant \operatorname{Pr}\left(e\left(G\left(n, p_{1}\right)\right) \geqslant N p_{1}+\omega \sqrt{N p_{1} q_{1}}\right) \leqslant{ }_{b} e^{-\omega^{2} / 2}, \\
& \operatorname{Pr}\left(e\left(G\left(n, p_{2}\right)\right)<m\right) \leqslant \operatorname{Pr}\left(e\left(G\left(n, p_{2}\right)\right) \leqslant N p_{2}-\omega \sqrt{N p_{2} q_{2}}\right) \leqslant{ }_{b} e^{-\omega^{2} / 2} .
\end{aligned}
$$


Since

$$
\operatorname{Pr}(S(G(n, p))>0)=\sum_{\mu=0}^{N} \operatorname{Pr}(e(G(n, p))=\mu) \operatorname{Pr}(S(G(n, \mu))>0),
$$

and $\operatorname{Pr}(S(G(n, \mu))>0)$ decreases with $\mu$, we have

$$
\begin{aligned}
\operatorname{Pr}\left(S\left(G\left(n, p_{1}\right)\right)>0\right) & \geqslant \operatorname{Pr}\left(e\left(G\left(n, p_{1}\right)\right) \leqslant m\right) \operatorname{Pr}(S(G(n, m))>0) \\
& \geqslant\left(1-O\left(e^{-\omega^{2} / 2}\right)\right) \operatorname{Pr}(S(G(n, m))>0),
\end{aligned}
$$

and

$$
\begin{aligned}
\operatorname{Pr}\left(S\left(G\left(n, p_{2}\right)\right)=0\right) & \left.\geqslant \operatorname{Pr}\left(e\left(G\left(n, p_{2}\right)\right) \geqslant m\right) \operatorname{Pr}(S(G(n, m))=0)\right) \\
& =\left(1-O\left(e^{-\omega^{2} / 2}\right)\right) \operatorname{Pr}(S(G(n, m))=0) .
\end{aligned}
$$

Therefore

$$
\begin{aligned}
\frac{\operatorname{Pr}\left(S\left(G\left(n, p_{1}\right)\right)>0\right)}{1-O\left(e^{-\omega^{2}}\right)} \geqslant & \operatorname{Pr}(S(G(n, m))>0) \\
& \geqslant \frac{\operatorname{Pr}\left(S\left(G\left(n, p_{2}\right)\right)>0\right)-O\left(e^{-\omega^{2} / 2}\right)}{1-O\left(e^{-\omega^{2} / 2}\right)} .
\end{aligned}
$$

Now, by (3.0.4),

$$
\begin{aligned}
& p_{1,2}=\frac{m}{N}\left(1+O\left(\omega m^{-1 / 2}\right)\right)=\frac{1+\lambda_{1,2} n^{-1 / 3}}{n}, \\
& \lambda_{1,2}=\lambda+O\left(\omega m^{-1 / 2}+n^{-2 / 3}\right)
\end{aligned}
$$

so, as $|\lambda|=o\left(n^{1 / 12}\right)$,

$$
\lambda_{1,2}^{3}=\lambda^{3}+O\left[\lambda^{2}\left(\omega m^{-1 / 2}+n^{-2 / 3}\right)\right]+O\left[\left(\omega m^{-1 / 2}\right)^{3}+n^{-2}\right]=\lambda^{3}+o\left(\omega n^{-1 / 3}\right) .
$$

That is, $\lambda_{1,2}^{3}-\lambda^{3} \rightarrow 0$. Hence,

$$
\operatorname{Pr}\left(S\left(G\left(n, p_{1,2}\right)\right)>0\right) \sim c(\lambda) n^{-1 / 12}
$$

Also $\omega^{2} \gg|\lambda|^{3}$ if $\omega^{1 / 8}$, which is compatible with the restriction $\omega \leqslant n^{1 / 6}$. For this choice of $\omega$, the relations $(3.0 .7)-(3.0 .8)$ imply: for $\lambda=o\left(n^{1 / 12}\right)$,

$$
\operatorname{Pr}(S(G(n, m))>0) \sim c(\lambda) n^{-1 / 12} .
$$

This completes the proof of Theorem 1.1 for $\hat{p}=1 / 2$. 


\section{Solvability (2-colorability) probability: $G(n, p), G(n, m)$ and $\hat{p}=1$.}

Consider the $G(n, p)$ case. We know that the system

$$
x_{i}+x_{j} \equiv 1(\bmod 2), \quad(i, j) \in E(G),
$$

is solvable iff the graph $G$ has no odd cycles. So a counterpart of (2.1.11) is

$$
\operatorname{Pr}\left(S_{n}>0, \mathcal{E}_{n} \leqslant L\right)=N(n, p)\left[x^{n}\right] \exp \left[\sum_{\ell=-1}^{L}\left(\frac{p}{q}\right)^{\ell} C_{\ell}^{e}(x)\right]
$$

$\left(S_{n}=S(G(n, p)), \mathcal{E}_{n}=\mathcal{E}(G(n, p))\right)$, where $C_{\ell}^{e}(x)$ is the exponential generating function of graphs $G$ without odd cycles, with an excess $\mathcal{E}(G)=\ell$. And again the events $\left\{S_{n}>0\right\}$ and $\left\{\mathcal{E}_{n} \leqslant L\right\}$ are positively correlated, i. e.

$$
\operatorname{Pr}\left(S_{n}>0, \mathcal{E}_{n} \leqslant L\right) \leqslant \operatorname{Pr}\left(S_{n}>0\right) \leqslant \frac{\operatorname{Pr}\left(S_{n}>0, \mathcal{E}_{n} \leqslant L\right)}{\operatorname{Pr}\left(\mathcal{E}_{n} \leqslant L\right)} .
$$

Thus the generating functions $C_{\ell}^{e}(x)$ take a center stage. Obviously

$$
C_{-1}^{e}(x)=C_{-1}(x)\left(=T(x)-\frac{1}{2} T^{2}(x)\right) .
$$

Furthermore, while

$$
C_{0}(x)=\frac{1}{2}\left(\ln \frac{1}{1-T(x)}-T(x)-\frac{1}{2} T^{2}(x)\right)
$$

for $C_{0}^{e}(x)$ we have

$$
C_{0}^{e}(x)=\frac{1}{4}\left(\ln \frac{1}{1-T^{2}(x)}-T^{2}(x)\right) .
$$

Indeed, we enumerate the connected unicyclic graphs with an even cycle, i. e. forests of an even number of rooted trees, whose roots form an undirected cycle. So

$$
C_{0}^{e}(x)=\sum_{\text {even } j \geqslant 4} \frac{(j-1) !}{2} \frac{T^{j}(x)}{j !},
$$

which simplifies to (4.0.2). Comparing $C_{0}^{e}(c)$ and $C_{0}(x)$ we see that, for $|x|<e^{-1}, x \rightarrow 1$, i. e. for $x$ dominant asymptotically,

$$
C_{0}^{e}(x)=\frac{1}{2} C_{0}(x)+\frac{1}{8}-\frac{1}{4} \ln 2+O(|T(x)-1|) ;
$$

in particular, $C_{0}^{e}(x) \sim(1 / 2) C_{0}(x)$. We want to show that this pattern persists for $\ell>0$, namely

$$
C_{\ell}^{e}(x) \sim \frac{1}{2^{\ell+1}} C_{\ell}(x), \quad\left(|x|<e^{-1}, x \rightarrow e^{-1}\right) .
$$


Comparing (2.1.11) and (4.0.1), and recalling the different roles played by $C_{0}(x)$ and $\left\{C_{\ell}(x)\right\}_{\ell>0}$ in the analysis of the $\hat{p}=1 / 2$ makes it transparent, hopefully, that for $\hat{p}=1$ we should have

$$
\operatorname{Pr}(G(n, p) \text { is 2-colorable })=\operatorname{Pr}\left(S_{n}>0\right) \sim 2^{-1 / 4} e^{1 / 8} c(\lambda) n^{-1 / 12} .
$$

Let us prove (4.0.4). First

Proposition 4.1. Given $n$ and $m \leqslant N:=\left(\begin{array}{l}n \\ 2\end{array}\right)$, let $C(n, m)$ denote the total number of connected graphs on $[n]$ with $m$ edges, and let $C^{e}(n, m)$ denote the total number of connected graphs without odd cycles. Then

$$
C^{e}(n, m) \leqslant \frac{1}{2^{m+1-n}} C(n, m) .
$$

Consequently

$$
C_{\ell}^{e}(x) \leqslant c \frac{1}{2^{\ell+1}} C_{\ell}(x), \quad \ell \geqslant-1 .
$$

Proof of Proposition 4.1. We begin with a simple claim.

Lemma 4.1. Let $T$ be a tree on the vertex set $[n]$. Let $X(T)$ denote the total number of paths in $T$ of an even edge-length 2 at least. Then $X(T) \geqslant X\left(P_{n}\right)$, where $P_{n}$ is a path on $[n]$, and

$$
X\left(P_{n}\right)=\left\lceil\frac{n(n-2)}{4}\right\rceil .
$$

Proof of Lemma 4.1. Pick a vertex $v \in[n]$, and introduce $V_{0}(T)$ and $V_{1}(T)$ the set of vertices reachable from $v$ by paths of even length 2 at least, and odd length respectively; in particular $v \in V_{0}$. Now every two vertices from $V_{i}(T),(i=0,1)$, are connected by an even path, while there is no even path connecting $v_{0} \in V_{0}(T)$ and $v_{1} \in V_{1}(T)$. Hence

$$
X(T)=\left(\begin{array}{c}
\left|V_{0}(T)\right| \\
2
\end{array}\right)+\left(\begin{array}{c}
\left|V_{1}(T)\right| \\
2
\end{array}\right) .
$$

It follows that $X(T)$ attains its minimum when $\left|V_{0}(T)\right|=\lfloor n / 2\rfloor$ and $\left|V_{1}(T)\right|=\lceil n / 2\rceil$, or the other way around, i. e. when $T=P_{n}$, and the minimum value is

$$
X\left(P_{n}\right)=\left(\begin{array}{c}
\lfloor n / 2\rfloor \\
2
\end{array}\right)+\left(\begin{array}{c}
\lceil n / 2\rceil \\
2
\end{array}\right)=\left\lceil\frac{n(n-2)}{4}\right\rceil .
$$

Armed with this Lemma, we will derive a recurrence inequality for $C^{e}(n, m)$. First we recall a recurrence equality for $C(n, m)$, [25], [3]: for $n \geqslant 3, n-1 \leqslant m \leqslant N$,

$$
\begin{aligned}
m C(n, m)= & (N-m+1) C(n, m-1) \\
& +\frac{1}{2} \sum_{\substack{n_{1}+n_{2}, m_{1}+m_{2}=m-1}}\left(\begin{array}{c}
n \\
n_{1}
\end{array}\right) n_{1} n_{2} C\left(n_{1}, m_{1}\right) C\left(n_{2}, m_{2}\right) .
\end{aligned}
$$


Explanation. The left hand side of (4.0.8) is the total number of the connected $(n, m)$ graphs with a marked edge. Each one of these graphs with a marked edge can be obtained in one of two, mutually exclusive ways. First way is inserting a marked edge into a connected graph on $[n]$ with $m-1$ edges, which accounts for the first term on the right hand side of (4.0.8); indeed $N-m+1$ is the total number of unordered pairs of vertices not connected by an edge in a given connected graph with $m-1$ edges. Second way is to start with a connected $\left(n_{1}, m_{1}\right)$ graph and a connected $\left(n_{2}, m_{2}\right)$ graph, having $m-1$ edges in total, and to add a marked edge that joins two connected graphs; $n_{1} n_{2}$ is the total number of ways to select two "contact" points, representing each of two graphs.

Let us see if there is a similar recursive formula for $C^{e}(n, m)$. Clearly, if a marked edge joins two connected graphs, none of these two graphs may have an odd cycle. So we definitely have the " $C^{e}(\cdot, \cdot)$ " counterpart of the second term on the right hand side of (4.0.8). As for a potential counterpart of the first term, a difficulty is that an additional $m$-th edge is not allowed to form an odd cycle with any of the $m-1$ edges already present. And so the total number of admissible options depends on the structure of a $(n, m-1)$ graph $G$ in question. (For such a graph to be connected, it is necessary that $m \geqslant n$.) However we can bound the number of options. $G$ is spanned by a tree $T$ on $[n]$, and none of the $m-1-(n-1)=m-n$ edges of $G \backslash T$ completes an odd cycle by joining the ends of an even path in $T$. By Lemma 4.1, the total number of those even paths is $\lceil n(n-2) / 4\rceil$, at least. Hence the total number of options for the $m$-edge is $N-(m-1)-\lceil n(n-2) / 4\rceil$, at most. And it is straightforward that, for $n \geqslant 3$ and by $m \geqslant n$,

$$
N-(m-1)-\left\lceil\frac{n(n-2)}{4}\right\rceil \leqslant \frac{1}{2}(N-(m-1)) .
$$

So $C^{e}(\cdot, \cdot)$ satisfies a recursive inequality: for $n \geqslant 3, n-1 \leqslant m \leqslant N$,

$$
\begin{aligned}
m C^{e}(n, m) \leqslant & \frac{1}{2}(N-m+1) C^{e}(n, m-1) \\
& +\frac{1}{2} \sum_{\substack{n_{1}+n_{2}, m_{1}+m_{2}=m-1}}\left(\begin{array}{c}
n \\
n_{1}
\end{array}\right) n_{1} n_{2} C^{e}\left(n_{1}, m_{1}\right) C^{e}\left(n_{2}, m_{2}\right) .
\end{aligned}
$$

$\left(C^{e}(\nu, \mu):=0\right.$ if $\nu=0$, or $\mu \notin\left[\nu-1,\left(\begin{array}{l}\nu \\ 2\end{array}\right)\right]$.) We will use (4.0.9) and induction to prove the bound (4.0.5). To this end, we define a lexicographical order, $\prec$, on $\{(n, m): n \geqslant$ $\left.1, n-1 \leqslant m \leqslant\left(\begin{array}{l}n \\ 2\end{array}\right)\right\}$ as follows: denoting $\ell=m-n$,

$$
\left(n_{1}, m_{1}\right) \prec\left(n_{2}, m_{2}\right) \Longleftrightarrow \ell_{1}<\ell_{2} \text {, or } \ell_{1}=\ell_{2} \text { and } n_{1}<n_{2} \text {. }
$$

The order $\prec$ is total, and $(1,0)$ is the minimal element. The inductive basis holds, since $C^{e}(1,0)=C(1,0)=1$, and $C^{e}(2,1)=C(2,1)=1$. Suppose that, for some $n \geqslant 2$ and $m \in\left[n-1,\left(\begin{array}{l}n \\ 2\end{array}\right)\right]$,

$$
C^{e}(\nu, \mu) \leqslant \frac{1}{2^{\mu-\nu+1}} C(\nu, \mu), \quad \forall(\nu, \mu) \prec(n, m) .
$$


Since $(n, m-1) \prec(n, m)$, the inductive assumption implies that

$$
\begin{aligned}
\frac{1}{2}(N-m+1) C^{e}(n, m-1) & \leqslant \frac{1}{2}(N-m+1) \frac{1}{2^{m-1-n+1}} C(n, m-1) \\
& =\frac{1}{2^{m-n+1}}(N-m+1) C(n, m) .
\end{aligned}
$$

Further, for the double sum in (4.0.9),

$$
m_{1}-n_{1}+1 \geqslant 0, \quad m_{2}-n_{2}+1 \geqslant 0,
$$

and

$$
\left(m_{1}-n_{1}+1\right)+\left(m_{2}-n_{2}+1\right)=m-1-n+2=m-n+1,
$$

so that

$$
m_{i}-n_{i}+1 \leqslant m-n+1 \Longrightarrow m_{i}-n_{i} \leqslant m-n, \quad i=1,2 .
$$

So, for $n_{1}, n_{2}>0$, we have $\left(n_{i}, m_{i}\right) \prec(n, m)$ and therefore, by the inductive assumption,

$$
\prod_{i=1}^{2} C^{e}\left(n_{i}, m_{i}\right) \leqslant \prod_{i=1}^{2} \frac{1}{2^{m_{i}-n_{i}+1}} C\left(n_{i}, m_{i}\right)=\frac{1}{2^{m-n+1}} \prod_{i=1}^{2} C\left(n_{i}, m_{i}\right) .
$$

Combining (4.0.9)-(4.0.11), and the recurrence equation (4.0.8) for $C(\cdot, \cdot)$, we obtain

$$
\begin{aligned}
m C^{e}(n, m) \leqslant & \frac{1}{2^{m-n+1}}(N-m+1) C(n, m-1) \\
& +\frac{1}{2^{m-n+1}} \frac{1}{2} \sum_{\substack{n_{1}+n_{2}, m_{1}+m_{2}=m-1}}\left(\begin{array}{c}
n \\
n_{1}
\end{array}\right) \prod_{i=1}^{2} n_{i} C\left(n_{i}, m_{i}\right) \\
= & \frac{1}{2^{m-n+1}} m C(n, m) .
\end{aligned}
$$

Thus the bound (4.0.3) holds for $(n, m)$ too. The proof of Proposition 4.1 is complete.

By Proposition 4.1 and and the formula (4.0.1), we have

$$
\begin{aligned}
& \operatorname{Pr}\left(S_{n}>0, \mathcal{E}_{n} \leqslant L\right) \\
\leqslant & N(n, p)\left[x^{n}\right] \exp \left[\frac{q}{p} C_{1}(x)+C_{0}^{e}(x)+\frac{1}{2} \sum_{\ell=1}^{L}\left(\frac{p}{2 q}\right)^{\ell} C_{\ell}(x)\right] .
\end{aligned}
$$

Since $C_{0}^{e}(x)$ is asymptotic to $(1 / 2) C_{0}(x)+\ln \left(2^{-1 / 4} e^{1 / 8}\right)$ as $x \rightarrow e^{-1}$, only a trivial change in the proof of Theorem 1.1 (i) is needed to show that

$$
\operatorname{Pr}\left(S_{n}>0\right) \lesssim 2^{-1 / 4} e^{1 / 8} c(\lambda) n^{-1 / 12}, \quad\left(|\lambda|=o\left(n^{1 / 12}\right) .\right.
$$

We omit the details. Furthermore, since for $\lambda \rightarrow-\infty$ we use $L=0$, the sums $\sum_{\ell=1}^{L}$ in (4.0.1), (4.0.12) disappear, and we obtain an asymptotic equality

$$
\operatorname{Pr}\left(S_{n}>0\right) \sim 2^{-1 / 4} e^{1 / 8} c(\lambda) n^{-1 / 12}, \quad\left(|\lambda|=o\left(n^{1 / 12}, \lambda \rightarrow-\infty\right)\right.
$$


To complete the proof of (ii), (case $\lambda=O(1)$ ), we need to prove (4.0.4) for each fixed $\ell>0$. Recall Wright's formula

$$
C_{\ell}(x)=(1-T(x))^{-3 \ell}\left[\sum_{d=0}^{2 \ell} c_{\ell, d}(1-T(x))^{d}\right], \quad(\ell>0) .
$$

Let us find a similar formula for $C_{\ell}^{e}(x), \ell>0$.

Proposition 4.2. For $\ell>0$,

$$
C_{\ell}^{e}(x)=\left(1-T^{2}(x)\right)^{-3 \ell}\left[\sum_{d=0}^{8 \ell-1} c_{\ell, d}^{e}(1-T(x))^{d}\right]
$$

where

$$
c_{\ell, 0}^{e}=2^{2 \ell-1} c_{\ell, 0}
$$

Consequently, for $|x|<e^{-1}$ and $x \rightarrow e^{-1}$,

$$
C_{\ell}^{e}(x) \sim \frac{1}{2^{\ell+1}} C_{\ell}(x)
$$

Proof of Proposition 4.2. We use the ideas of Wright's original proof of (4.0.14), and the improvements suggested by Stepanov [24], (cf. [13], Section 9).

Given a connected graph $G$ on $[n]$, with an excess $\ell=e(G)-v(G)>0$, we apply a "pruning" algorithm which successively deletes vertices of degree 1. Obviously the excess is preserved, and so for a terminal graph (core) $\bar{G}$ we have $e(\bar{G})-v(\bar{G})=\ell$. $\bar{G}$ inherits all the cycles of $G$, and thus $\bar{G}$ has only even cycles iff $G$ does. A minimum degree of $\bar{G}$ is 2 at least, and - since $\ell(\bar{G})=\ell>0$ - a maximum degree is 3 at least. Next we apply a "cancellation" algorithm to $\bar{G}$ : at each step, we delete a vertex of degree 2 , splicing together the two edges it formerly touched. The excess is preserved again. Once all the vertices of degree 2 are gone, we get a connected multigraph (kernel) $\tilde{G}$, with possible loops and parallel edges, and a minimum vertex degree 3 at least. Thus

$$
2 e(\tilde{G}) \geqslant 3 v(\tilde{G}), \quad e(\tilde{G})-v(\tilde{G})=\ell
$$

and so

$$
v(\tilde{G}) \leqslant 2 \ell, \quad e(\tilde{G}) \leqslant 3 \ell .
$$

Notice that the largest numbers of vertices and the edges in the kernel are $2 \ell$ and $3 \ell$ respectively, and the corresponding kernel is a 3-regular multigraph. (In [13] graphs $G$ with such kernels were called clean. It is these clean graphs that are most populous asymptotically among all connected graphs on $[n]$ with excess $\ell$.) Now that we have a reduced number $v(\tilde{G})$ of vertices, we relabel them using indices from $[v(\tilde{G})]$ and preserving the order of their old indices from $[n]$. Under this rule, it follows that the number of kernels $\tilde{G}$ for the collection of all connected graphs $G$ on $[n]$ with excess $\ell$ is a function of $\ell$ only! 
A key element of Wright's argument was the following identity. Let $M$ be a connected multigraph on a vertex set $[\nu]$, with $\mu_{i}$ indistinguishable loops at vertex $i$, and $\mu_{i j}$ indistinguishable parallel edges joining $i$ and $j,(i, j \in[\nu], i \neq j)$. Let $h_{n, M}$ denote the total number of the connected simple graphs $G$ on $[n]$, with minimum degree 2 at least and maximum degree 3 at least (core-type graphs, in short), such that $\tilde{G}=M$. Letting

$$
\mathcal{H}_{M}(z)=\sum_{n} \frac{h_{n, M}}{n !} z^{n}
$$

we have

$$
\mathcal{H}_{M}(z)=\frac{\kappa}{\nu !} \frac{z^{\nu}}{(1-z)^{\mu}} \cdot \mathcal{K}_{M}(z), \quad \mu:=\sum_{i} \mu_{i}+\sum_{i<j} \mu_{i j}
$$

where

$$
\begin{aligned}
\mathcal{K}_{M}(z) & =\prod_{1 \leqslant i \leqslant \nu}\left(z^{2 \mu_{i}} \prod_{1 \leqslant i<j \leqslant \nu} z^{\mu_{i j}-1}\left(\mu_{i j}-\left(\mu_{i j}-1\right) z\right)\right), \\
\kappa & =\prod_{1 \leqslant i \leqslant \nu} \frac{1}{2^{\mu_{i}} \mu_{i} !} \prod_{1 \leqslant i<j \leqslant \nu} \frac{1}{\mu_{i j} !} .
\end{aligned}
$$

(Observe that $\mathcal{K}_{M}(1)=1$.) Once (4.0.19)-(4.0.20) are established, it is easy to determine $H_{M}(x)$, the exponential generating function of all connected graphs $G$ whose kernel is the multigraph $M$. Indeed to go from a core $\bar{G}$ back to $G$ on $[n]$ we need to choose an ordered sequence of $v(\bar{G})$ of rooted trees, of total size $n$, and plant them at the vertices of $\bar{G}$, moving increasingly from vertex 1 to vertex $v(\bar{G})$. Since the generating function of such sequences is $T(x)^{v(\bar{G})}$, we see that

$$
H_{M}(x)=\sum_{n} \frac{h_{n, M}}{n !} T(x)^{n}=\mathcal{H}_{M}(T(x)) .
$$

Finally

$$
\begin{aligned}
C_{\ell}(x) & =\sum_{M: e(M)-v(M)=\ell} H_{M}(x)=(1-T(x))^{-3 \ell}\left[\sum_{d=0}^{2 \ell} c_{\ell, d}(1-T(x))^{d}\right] ; \\
c_{\ell, 0} & :=\frac{1}{(2 \ell) !} \sum_{\boldsymbol{\mu} \text { meets }(4.0 .18)} \kappa(\boldsymbol{\mu}) .
\end{aligned}
$$

Our first step is to obtain a counterpart of (4.0.18)-(4.0.20) for

$$
\mathcal{H}_{M}^{e}(z)=\sum_{n} \frac{h_{n, M}^{e}}{n !} z^{n}
$$

where $h_{n, M}^{e}$ is the total number of the connected core-type graphs on $[n]$ with only even cycles, that cancel to a given multigraph $M$. To this end, consider an auxilliary problem. Let

$$
\mu_{i}=\mu_{i}^{e}+\mu_{i}^{o}, \quad \mu_{i j}=\mu_{i j}^{e}+\mu_{i j}^{o}, \quad(1 \leqslant i \neq j \leqslant \nu)
$$


Let $h_{n,\left(\boldsymbol{\mu}^{e}, \boldsymbol{\mu}^{o}\right)}$ denote the total number of the core-type graphs $G$ on $[n]$, which cancel to $M$, such that: (1) for each $i, G$ has an even (odd resp.) number of 2-degree vertices put on each of $\mu_{i}^{e}$ ( $\mu_{i}^{0}$ resp.) loops at vertex $i$ of $M$; (2) for each $(i, j), G$ has an even (odd resp.) number of 2-degree vertices put on each of $\mu_{i j}^{e}$ ( $\mu_{i j}^{o}$ resp.) parallel edges joining the vertices $i$ and $j$ in $M$. Let us determine

$$
\mathcal{H}_{\left(\boldsymbol{\mu}^{e}, \boldsymbol{\mu}^{o}\right)}(z)=\sum_{n} \frac{h_{n,\left(\boldsymbol{\mu}^{e}, \boldsymbol{\mu}^{o}\right)}}{n !} z^{n} .
$$

A core-type graph $G$ on $[n]$ cancelling to $M$ and meeting the parity conditions (1)-(2) can be viewed as a partition of $[n]$ into:

(a) a subset of cardinality $\nu$, whose elements are the assigned to the $\nu$ vertices of $M$ in a unique (order-preserving) fashion;

(b) $\forall i \in[\nu]$, a collection of $\mu_{i}$ ordered subsets, each having 2 elements at least (as $G$ is simple), such that exactly $\mu_{i}^{e}$ ( $\mu_{i}^{o}$ resp.) subsets have an even (odd resp.) number of elements;

(c) $\forall 1 \leqslant i \neq j \leqslant \nu$, a collection of $\mu_{i j}$ ordered subsets, with at most one empty subset (as $G$ is simple), such that exactly $\mu_{i j}^{e}$ ( $\mu_{i j}^{o}$ resp.) subsets have an even (odd resp.) number of elements.

So $\mathcal{H}_{\left(\boldsymbol{\mu}^{e}, \boldsymbol{\mu}^{o}\right)}(z)$ is the product of generating functions $\mathcal{H}_{t}(z)$ corresponding to $1+\nu+\left(\begin{array}{c}\nu \\ 2\end{array}\right)$ combinatorial structures described in (a), (b), (c). The first is easy:

$$
\mathcal{H}(z)=\frac{z^{\nu}}{\nu !}
$$

Next, for $i \in[\nu]$,

$$
\mathcal{H}_{i}(z)=\frac{1}{2^{\mu_{i}} \mu_{i}^{e} ! \mu_{i}^{o !}} \sum_{n} a_{n,\left(\mu_{i}^{e}, \mu_{i}^{o}\right)} z^{n} ;
$$

here $a_{n,\left(\mu_{i}^{e}, \mu_{i}^{o}\right)}$ is the total number of compositions of $n$ with $\mu_{i}$ parts, each 2 at least, such that the first $\mu_{i}^{e}$ parts (the last $\mu_{i}^{0}$ parts resp.) are even (odd resp.). The factor $1 / 2^{\mu_{i}}$ is needed as we do not distinguish between two opposite orderings of vertices sprinkled on each of $\mu_{i}$ loops of $M$ at $i$. Consequently

$$
\begin{aligned}
\mathcal{H}_{i}(z) & =\frac{1}{2^{\mu_{i}} \mu_{i}^{e} ! \mu_{i}^{o} !}\left(\sum_{k \geqslant 1} z^{2 k}\right)^{\mu_{i}^{e}}\left(\sum_{k \geqslant 1} z^{2 k+1}\right)^{\mu_{i}^{o}} \\
& =\frac{1}{2^{\mu_{i}} \mu_{i}^{e} ! \mu_{i}^{o} !} \frac{z^{2 \mu_{i}+\mu_{i}^{o}}}{\left(1-z^{2}\right)^{\mu_{i}}} .
\end{aligned}
$$

Similarly, for each $1 \leqslant i<j \leqslant \nu$,

$$
\mathcal{H}_{i j}(z)=\frac{1}{\mu_{i j}^{e} ! \mu_{i j}^{o} !}\left[\left(\frac{z^{2}}{1-z^{2}}\right)^{\mu_{i j}^{e}}+\mu_{i j}^{e}\left(\frac{z^{2}}{1-z^{2}}\right)^{\mu_{i j}^{e}-1}\right]\left(\frac{z}{1-z^{2}}\right)^{\mu_{i j}^{o}} .
$$


Taking the product of the generating functions in (4.0.24)-(4.0.26) we obtain

$$
\mathcal{H}_{\left(\boldsymbol{\mu}^{e}, \boldsymbol{\mu}^{o}\right)}(z)=\frac{\kappa}{\nu !} \frac{z^{\nu}}{\left(1-z^{2}\right)^{\mu}} \cdot \mathcal{K}_{\left(\boldsymbol{\mu}^{e}, \boldsymbol{\mu}^{o}\right)}(z)
$$

where

$$
\mathcal{K}_{\left(\boldsymbol{\mu}^{e}, \boldsymbol{\mu}^{o}\right)}(z)=\prod_{i \in[\nu]}\left(\begin{array}{c}
\mu_{i} \\
\mu_{i}^{e}
\end{array}\right) z^{2 \mu_{i}+\mu_{i}^{o}} \cdot \prod_{1 \leqslant i<j \leqslant \nu}\left(\begin{array}{c}
\mu_{i j} \\
\mu_{i j}^{e}
\end{array}\right) z^{\mu_{i j}+\mu_{i j}^{e}-2}\left[\mu_{i j}^{e}-\left(\mu_{i j}^{e}-1\right) z^{2}\right] .
$$

As a partial check, summing over $\left(\boldsymbol{\mu}^{e}, \boldsymbol{\mu}^{o}\right)$, we obtain Wright's formula (4.0.18)-(4.0.20).

Now, for a core-type graph $G$ on $[n]$ without odd cycles, that cancels to $M, G$ 's parity parameters $\boldsymbol{\mu}^{e}, \boldsymbol{\mu}^{o}$ must satisfy certain conditions. First of all, for each $i \in[\nu], \mu_{i}^{e}=0$, since otherwise $G$ would have an odd cycle, with a single branching vertex. Likewise, for $\mu_{i j}>0$, the numbers of 2-degree vertices of $G$ on $\mu_{i j}$ parallel edges of $M$ must all be of the same parity, hence $\mu_{i j}^{e}=\mu_{i j}$ or $\mu_{i j}^{o}=\mu_{i j}$. Subject to this condition, how many choices for $\left(\boldsymbol{\mu}^{e}, \boldsymbol{\mu}^{o}\right)$ do we have? For each $(i, j)$ such that $\mu_{i j}>0$, define

$$
b_{i j}=b_{j i}= \begin{cases}1, & \text { if } \mu_{i j}^{e}=\mu_{i j} \\ 0, & \text { if } \mu_{i j}^{e}=0\end{cases}
$$

If $C$ is a cycle in $M$, then the parity of a cycle in $G$ that cancels to $C$ is the parity of $b(C):=\sum_{(i, j) \in C} b_{i j}$. Hence $b(C)$ must be even for all cycles $C$, and we need to check this condition only for simple cycles that do not use parallel edges. Let $T=T(M)$ be a tree on $[\nu]$ that spans $M$. Pick $\mu_{i j}^{e}$ for all $\nu-1$ pairs $(i, j)$ such that $(i, j) \in E(T)$, i. e. one of $\mu_{i j}$ parallel edges is in $E(T)$. Let $\mu_{i j}>0$ and $e=(i, j) \notin E(T)$. Then $e$ completes a cycle $C$ with a path in $T$ that connects $i$ and $j$. The condition " $b(C)$ is even" determines $\mu_{i j}^{e}$ uniquely. Hence a choice of $\nu-1$ values of $\mu_{i j}^{e}$ determines uniquely the remaining $\mu_{. .}^{e}$. Arguing as in the proof of Lemma 2.1.1, we see that the condition "b(C) is even" will hold for all other cycles $C$. Thus we have have $2^{\nu-1}$ choices for $\left(\boldsymbol{\mu}^{e}, \boldsymbol{\mu}^{o}\right)$.

For each of those choices, (4.0.28) becomes

$$
\mathcal{K}_{\left(\boldsymbol{\mu}^{e}, \boldsymbol{\mu}^{o}\right)}(z)=\prod_{i \in[\nu]} z^{3 \mu_{i}} \cdot \prod_{\substack{1 \leqslant i<j \leqslant \nu \\ \mu_{i j}^{i}=\mu_{i j}}} z^{2\left(\mu_{i j}-1\right)}\left[\mu_{i j}-\left(\mu_{i j}-1\right) z^{2}\right] \cdot \prod_{\substack{1 \leqslant i<j \leqslant \nu \\ \mu_{i j}=0}} z^{\mu_{i j}} .
$$

For each of these $2^{\nu-1}$ polynomials,

$$
\mathcal{K}_{\left(\boldsymbol{\mu}^{e}, \boldsymbol{\mu}^{\circ}\right)}(1)=1,
$$

and

$$
\operatorname{deg} \mathcal{K}_{\left(\boldsymbol{\mu}^{e}, \boldsymbol{\mu}^{\circ}\right)}(z) \leqslant 3 \sum_{i} \mu_{i}+2 \sum_{1 \leqslant i<j \leqslant \nu} \mu_{i j} .
$$

Using the constraints

$$
\sum_{i} \mu_{i}+\sum_{1 \leqslant i<j \leqslant \nu} \mu_{i j}=\mu, \quad \sum_{i} \mu_{i}+2 \sum_{1 \leqslant i<j \leqslant \nu} \mu_{i j} \geqslant 3 \nu,
$$


we easily obtain then that

$$
\operatorname{deg} \mathcal{K}_{\left(\boldsymbol{\mu}^{e}, \boldsymbol{\mu}^{o}\right)}(z) \leqslant 4 \mu-3 \nu
$$

Now, the generating function $\mathcal{H}_{M}^{e}(z)$ of the core-type graphs $G$ without odd cycles that cancel to $M$ is the sum of $\mathcal{H}_{\left(\boldsymbol{\mu}^{e}, \boldsymbol{\mu}^{o}\right)}(z)$ over all $2^{\nu-1}$ sets of feasible pairs $\left(\boldsymbol{\mu}^{e}, \boldsymbol{\mu}^{o}\right)$. Using (4.0.27), (4.0.29) and (4.0.30) we arrive at the following formula.

Lemma 4.2. For each kernel $M$, with $\ell:=\mu-\nu>0$,

$$
\mathcal{H}_{M}^{e}(z)=\frac{\kappa}{\nu !} \frac{2^{\nu-1}}{\left(1-z^{2}\right)^{\mu}} P_{M}(z)
$$

where $P_{M}(z)$ is a polynomial of degree $4 \mu-3 \nu=\mu+3 \ell$ at most, and $P_{M}(1)=1$.

This Lemma directly implies

\section{Corollary 4.1.}

$$
C_{\ell}^{e}(x)=\sum_{M: e(M)-v(M)=\ell} H_{M}^{e}(x)
$$

where

$$
H_{M}^{e}(x)=\frac{\kappa}{\nu !} \frac{2^{\nu-1}}{\left(1-T^{2}(x)\right)^{\mu}} P_{M}(T(x))
$$

Using $\ell<\mu(M) \leqslant 3 \ell, \nu(M)=\mu(M)-\ell$, we deduce from (4.0.32)-(4.0.33) that

$$
C_{\ell}^{e}(x)=\left(1-T^{2}(x)\right)^{-3 \ell}\left[\sum_{d=0}^{8 \ell-1} c_{\ell, d}^{e}(1-T(x))^{d}\right],
$$

where

$$
c_{\ell, 0}^{e}=\frac{2^{2 \ell-1}}{(2 \ell) !} \sum_{\boldsymbol{\mu} \text { meets }(4.0 .19)} \kappa(\boldsymbol{\mu}) .
$$

So, by the second line in $(4.0 .22), c_{\ell, 0}^{e}=2^{2 \ell-1} c_{\ell, 0}$. The proof of Proposition 4.2 is complete.

Comparing (4.0.14) and (4.0.34)-(4.0.35), and using $T\left(e^{-1}\right)=1$, we obtain: for $\ell>0$,

$$
C_{\ell}^{e}(x)=\frac{1}{2^{\ell+1}} C_{\ell}(x)+O\left(|1-T(x)|^{-3 \ell+1}\right), \quad\left(|x|<e^{-1}, x \rightarrow e^{-1}\right) .
$$

And we recall, (4.0.3), that

$$
C_{0}^{e}(x)=\frac{1}{2} C_{0}(x)+\ln \left(2^{-1 / 4} e^{1 / 8}\right)+O(|T(x)-1|) .
$$

Now, by (4.0.1), for a fixed $L>0$,

$$
\operatorname{Pr}\left(S_{n}>0, \mathcal{E}_{n} \leqslant L\right)=N(n, p) \oint_{\Gamma} x^{-n-1} \exp \left[\sum_{\ell=-1}^{L}\left(\frac{p}{q}\right)^{\ell} C_{\ell}^{e}(x)\right] d x,
$$


where $\Gamma$ is within the disc $|x|<e^{-1}$. As in Section 2.3 , we switch to $y$ by $x=y e^{-y}$, and choose in the $y$-plane the circular contour $\Gamma^{\prime} y=e^{-a n^{-1 / 3}+i \theta}, a>0$ being fixed this time. Observe that, for each $1 \leqslant \ell \leqslant L$,

$$
\left(\frac{p}{q}\right)^{\ell}\left|1-T\left(y e^{-y}\right)\right|^{-3 \ell+1} \leqslant{ }_{b} n^{-\ell}|1-y|^{-3 \ell+1} \leqslant{ }_{b} n^{-1 / 3}
$$

and, likewise in (4.0.3) the remainder term $O\left(\left|T\left(y e^{-y}\right)-1\right|\right)$ is $O\left(n^{-1 / 3}\right)$. And of course $C_{-1}^{e}\left(y e^{-y}\right)=C_{-1}\left(y e^{-y}\right)$. On the basis of (4.0.36)-(4.0.37), it can be shown then that

$$
\begin{aligned}
\operatorname{Pr}\left(S_{n}\right. & \left.>0, \mathcal{E}_{n} \leqslant L\right) \\
& \sim 2^{-1 / 4} e^{1 / 8} N(n, p) \oint_{\Gamma^{\prime}}\left(y e^{-y}\right)^{-n-1} \exp \left[\frac{1}{2} \sum_{\ell=-1}^{L}\left(\frac{p}{2 q}\right)^{\ell} C_{\ell}\left(y e^{-y}\right)\right] d\left(y e^{-y}\right),
\end{aligned}
$$

where now $\Gamma^{\prime}$ can be replaced by a circular contour of an arbitrarily small radius. Going back to the $x$-plane, we recognize (see $(2.1 .11)$ ) the value of the resulting integral as

$$
\left.2^{-1 / 4} e^{1 / 8} \operatorname{Pr}\left(S_{n}>0, \mathcal{E}_{n} \leqslant L\right)\right|_{\hat{p}=1 / 2},
$$

for $\lambda=O(1)$ needless to say. By (2.1.13), the latter probability is at least

$$
\left.\operatorname{Pr}\left(S_{n}>0\right)\right|_{\hat{p}=1 / 2} \cdot \operatorname{Pr}\left(\mathcal{E}_{n} \leqslant L\right) .
$$

Letting $n \rightarrow \infty$, and using the part (i) for $\left.\operatorname{Pr}\left(S_{n}>0\right)\right|_{\hat{p}=1 / 2}$, we get

$$
\liminf \frac{\operatorname{Pr}\left(S_{n}>0, \mathcal{E}_{n} \leqslant L\right)}{2^{-1 / 4} e^{1 / 8} c(\lambda) n^{-1 / 12}} \geqslant \liminf \operatorname{Pr}\left(\mathcal{E}_{n} \leqslant L\right) .
$$

Since $\operatorname{Pr}\left(S_{n}>0\right) \geqslant \operatorname{Pr}\left(S_{n}>0, \mathcal{E}_{n} \leqslant L\right)$, and $\mathcal{E}_{n}=O_{P}(1)$, letting $L \uparrow \infty$ enables us to conclude that

$$
\operatorname{Pr}\left(S_{n}>0\right) \gtrsim 2^{-1 / 4} e^{1 / 8} c(\lambda) n^{-1 / 12} .
$$

Together with (4.0.14) this proves that

$$
\operatorname{Pr}\left(S_{n}>0\right) \sim 2^{-1 / 4} e^{1 / 8} c(\lambda) n^{-1 / 12} .
$$

The proof of Theorem 1.1 (ii) is now complete.

Acknowledgment. We are grateful to the participants of a graduate student workshop at Ohio State University for helpful commentaries during numerous discussions of various phases of this study. We thank Mike Molloy for posing a 2-colorability problem of the critical graph $G(n, m)$, and Greg Sorkin for an encouragement when we needed it most. Ákos Seres and Saleh Tanveer, members of the second author's Ph.D Committee, provided valuable comments on the project. A reviewer provided us with a series of helpful suggestions. Finally we must acknowledge our big debt to the Editors Michael Krivelevich, Brendan McKay and Ian Wanless for their thoughtful consideration. Brendan very generously volunteered to give a crucial help with typesetting the paper according to the Journal standards. 


\section{References}

[1] G. N. Bagaev, Random graphs with degree of connectedness 2 (Russian), Discret. Analiz, 22 (1973) 3-14.

[2] G. N. Bagaev and E. F. Dmitriev, Enumeration of connected labeled bipartite graphs (Russian), Dokl. Akad. Nauk BSSR,28 (1984) 1061-1063.

[3] E. A. Bender, E. R. Canfield and B. D. McKay, The asymptotic number of labeled connected graphs with a given number of vertices and edges, Random Structures and Algorithms, 1 (1990) 127-169.

[4] B. Bollobás, The evolution of random graphs, Trans. Amer. Math. Soc.,286 (1984) 257-274.

[5] B. Bollobás, Random Graphs, Second Edition Cambridge University Press, (2001).

[6] B. Bollobás, C. Borgs, J. T. Chayes, J. H. Kim and D. B. Wilson, The scaling window of the 2-SAT transition, Random Structures and Algorithms, 8 (2001) 201-256.

[7] V. Chvátal and B. Reed, Mick gets some (the odds are on his side), 33th Annual Symposium on Foundations of Computer Science, IEEE Comput. Soc. Press, Los Alamitos, CA, (1992) 620-627.

[8] D. Coppersmith, D. Gamarnik, M. T. Hajiaghayi and G. B. Sorkin, Random MAX SAT, random MAX CUT, and their phase transitions, Random Structures and Algorithms, 24 (2004) 502-545.

[9] N. Creignon and H. Daudé, Smooth and sharp thresholds for random $k$-XOR-CNF satisfiability, Theor. Inform. Appl., 37 (2003) 127-147.

[10] H. Daudé and V. Ravelomanana, Random 2-XORSAT at the satisfiability threshold, LATIN 2008: Theoretical Informatics, 8th Latin American Symposium Proceedings, (2008) 12-23.

[11] O. Dubois and J. Mandler, The 3-XORSAT Threshold, Proceedings of the 43rd Symposium on Foundations of Computer Science, (2002) 769 - 778.

[12] G. R. Grimmett and D. R. Stirzaker, Probability and Random Processes: Problems and Solutions, Clarendon Press, Oxford (1992).

[13] S. Janson, D. Knuth, T. Euczak and B. Pittel, The birth of the giant component, Random Structures and Algorithms, 4 (1993) 233-358.

[14] V. F. Kolchin, Random Graphs, Cambridge University Press, (1999).

[15] T. Euczak, Component behavior near the critical point of the random graph process, Random Structures and Algorithms, 1 (1990) 287-310.

[16] T. Łuczak, On the equivalence of two basic models of random graphs, Random Graphs '87 Poznań, Proceedings, Eds. M. Karoński, J. Jaworski and A. Ruciński, 151-158.

[17] T. Euczak, B. Pittel and J. Wierman, The structure of a random graph near the point of the phase transition, Trans. Amer. Math. Soc., 341 (1994) 721-748. 
[18] P. D. Miller, Applied Asymptotic Analysis, Providence, RI: American Mathematical Society , (2006).

[19] M. Molloy, Personal communication, (2007).

[20] J. W. Moon, Counting Labelled Trees, Canad. Math. Congress, Montreal, (1970).

[21] B. Pittel, A random graph with a subcritical number of edges, Trans. Amer. Math. Soc., 309 (1988) 51-75.

[22] B. Pittel, On the largest component of the random graph at a nearcritical stage, $J$. Comb. Theory B, 82 (2001) 237-269.

[23] A. D. Scott and G. B. Sorkin, Solving sparse random instances of MAX CUT and MAX 2-CSP in linear expected time, Combinatorics, Probability and Computing, 15 (2006) 281-315.

[24] V. E. Stepanov, On some feautures of the structure of a random graph near a critical point, Theory Probab. Appl., 32, (1988) 573-594.

[25] E. M. Wright, The number of connected sparsely edged graphs, J. Graph Theory, 1 (1977) 317-330.

[26] E. M. Wright, The number of connected sparsely edged graphs. III. Asymptotic results, J. Graph Theory, 4 (1980) 393-407.

\section{Appendix}

Proof of (2.3.22). (i) For the second bound, we use (2.3.19) and, setting $s=a+i t$,

$$
\left|s^{1-y} \exp \left(\frac{\mu s^{2}}{2}+\frac{s^{3}}{3}\right)\right| \leqslant a^{1-y} \exp \left(\frac{\mu a^{2}}{2}+\frac{a^{3}}{3}\right) \exp \left[-t^{2}(a+\mu / 2)\right] .
$$

So

$$
A(y, \mu) \leqslant(2 \pi)^{-1} \sqrt{\frac{\pi}{a+\mu / 2}} \exp \left(-\frac{\mu^{3}}{6}+\frac{\mu a^{2}}{2}+\frac{a^{3}}{3}\right) .
$$

(ii) For the first bound, we use (2.3.20), i. e.

$$
e^{\mu^{3} / 6} 3^{(y+1) / 3} A(y, \mu)=\sum_{k=0}^{\infty} \frac{\left(3^{2 / 3} \mu / 2\right)^{k}}{\Gamma(k+1) \Gamma((y+1-2 k) / 3)},
$$

and the inequalities

$$
\frac{(1 / 2)^{a+b+2}}{a+b+2} \leqslant \frac{\Gamma(a+1) \Gamma(b+1)}{\Gamma(a+b+2)} \leqslant \frac{a^{a} b^{b}}{(a+b)^{a+b}} \leqslant 1, \quad(a \geqslant 0, b \geqslant 0),
$$

which follow from a classic formula

$$
\int_{0}^{1} x^{a}(1-x)^{b} d x=\frac{\Gamma(a+1) \Gamma(b+1)}{\Gamma(a+b+2)},
$$

THE EleCtronic Journal of COMBinatorics 17 (2010), \#R92 
and

$$
\begin{aligned}
\max _{x \in[0,1]} x^{a}(1-x)^{b} & =\frac{a^{a} b^{b}}{(a+b)^{a+b}}, \\
\int_{0}^{1} x^{a}(1-x)^{b} d x & \geqslant(1 / 2)^{b} \int_{0}^{1 / 2} x^{a} d x+(1 / 2)^{a} \int_{1 / 2}^{1}(1-x)^{b} d x \\
& =(1 / 2)^{a+b+1}\left(\frac{1}{a+1}+\frac{1}{b+1}\right) .
\end{aligned}
$$

Break the sum in (A.1) into $\Sigma_{1}, \Sigma_{2}$, and $\Sigma_{3}$, for $\{k \geqslant 2:(y+1-2 k) / 3 \geqslant 1\}$, $\{k \geqslant 1:(y+1-2 k) / 3 \leqslant 0\}$, and $\{k=0,1:$ or $(y+1-2 k) / 3 \geqslant 1\}$, respectively. (Recall that $\Gamma(0)=\infty$.) For $\Sigma_{1}$,

$$
\begin{aligned}
\frac{1}{\Gamma(k+1) \Gamma((y+1-2 k) / 3))} & =\frac{\Gamma(2 k / 3)}{\Gamma(k+1)} \cdot \frac{1}{\Gamma((y+1-2 k) / 3) \Gamma(2 k / 3)} \\
& \leqslant \frac{2^{(y+1) / 3}}{\Gamma((y+1) / 3)} \cdot \frac{\Gamma(2 k / 3) \Gamma(k / 3+1)}{\Gamma(k+1)} \cdot \frac{1}{\Gamma(k / 3+1)} \\
& \leqslant \frac{2^{(y+1) / 3}}{\Gamma((y+1) / 3)} \cdot 2 \frac{(2 k / 3)^{2 k / 3-1}(k / 3)^{k / 3}}{k^{k-1}} \cdot \frac{1}{\Gamma(k / 3+1)} \\
& \leqslant 6 \frac{2^{(y+1) / 3}}{\Gamma((y+1) / 3)} \cdot \frac{\left(2^{2 / 3} / 3\right)^{k}}{\Gamma(k / 3+1)} .
\end{aligned}
$$

Therefore

$$
\left|\Sigma_{1}\right| \leqslant 6 \frac{2^{(y+1) / 3}}{\Gamma((y+1) / 3} \sum_{k \geqslant 0} \frac{\left(|\mu|^{3} / 6\right)^{k / 3}}{\Gamma(k / 3+1)} \leqslant b\left(|\mu|^{3} \vee 1\right) \frac{2^{(y+1) / 3}}{\Gamma((y+1) / 3)} \cdot e^{|\mu|^{3} / 6} .
$$

For $\Sigma_{2}$, we use

$$
\Gamma(z) \Gamma(1-z)=\frac{\pi}{\sin (\pi z)} \Longrightarrow \frac{1}{|\Gamma((y+1-2 k) / 3)|} \leqslant \Gamma(1+(2 k-y-1) / 3),
$$

and

$$
\begin{aligned}
& \frac{\Gamma(1+(2 k-y-1) / 3)}{\Gamma(k+1)} \leqslant \frac{1}{\Gamma(1+(y+1) / 3)} \cdot \frac{\Gamma(2 k / 3+2)}{\Gamma(k+1)} \\
= & \frac{1}{\Gamma(1+(y+1) / 3)} \cdot \frac{\Gamma(2 k / 3+2) \Gamma(k / 3+1)}{\Gamma(k+1)} \cdot \frac{1}{\Gamma(k / 3+1)} \\
\leqslant & \frac{1}{\Gamma(1+(y+1) / 3)} \cdot \frac{\Gamma(k+3)}{\Gamma(k+1)} \cdot \frac{(k / 3)^{k / 3}(1+2 k / 3)^{1+2 k / 3}}{(k+1)^{k+1}} \\
\leqslant & \frac{1}{\Gamma(1+(y+1) / 3)} \cdot(k+2)^{2} \frac{\left(2^{2 / 3} / 3\right)^{k}}{\Gamma(k / 3+1)} .
\end{aligned}
$$

Therefore

$$
\left|\Sigma_{2}\right| \leqslant{ }_{b}\left(|\mu|^{9} \vee 1\right) \frac{1}{\Gamma((y+1) / 3)} \cdot e^{|\mu|^{3} / 6}
$$


And it is not difficult to show that

$$
\left|\Sigma_{3}\right| \leqslant{ }_{b}\left|\Sigma_{1}\right|+\left|\Sigma_{2}\right|
$$

The relations (A.1)-(A.4) imply that

$$
A(y, \mu) \leqslant_{b} e^{|\mu|^{3} / 2} \frac{(2 / 3)^{\frac{y+1}{3}}}{\Gamma\left(\frac{y+1}{3}\right)} .
$$

Proof of (2.3.23)-(2.3.24). Again we use (2.3.19). We will choose $a=\xi$, where $\xi=\xi(y, \mu)$ is a maximum point of

$$
\Psi(a ; y, \mu):=-y \ln a+\frac{\mu a^{2}}{2}+\frac{a^{3}}{3}, \quad a \in(0, \infty),
$$

i. e. a positive root of

$$
\Psi_{a}^{(1)}(a ; y, \mu)=\mu a+a^{2}-\frac{y}{a}=0 .
$$

A root exists and is unique, since $\Psi_{a}(0+; y, \mu)=-\infty, \Psi(\infty ; y, \mu)=\infty$ and

$$
\Psi_{a}^{(2)}(a ; y, \mu)=\mu+2 a+\frac{y}{a^{2}}>0, \quad(a \geqslant 0) .
$$

Observe that $\mu \xi^{2} / y$ is bounded away from zero. If not, then, by (A.5),

$$
\frac{\mu^{3} \xi^{6}}{y^{3}} \rightarrow 0, \frac{y^{2}}{\xi^{6}} \rightarrow 1
$$

which implies that $\mu^{3} / y \rightarrow 0$, contradicting $y=O\left(\lambda^{3}\right)=O\left(\mu^{3}\right)$.

Break the integral in (2.3.19) into $I_{1}$ over $|t| \leqslant \mu^{-1 / 2} y^{1 / 7}$ and $I_{2}$ over $|t| \geqslant \mu^{-1 / 2} y^{1 / 7}$. Arguing as the part (i) of the previous proof, we bound

$$
\begin{aligned}
\left|I_{2}\right| & \leqslant b \exp [\Psi(\xi ; y, \mu)] \int_{|t| \geqslant \mu^{-1 / 2} y^{1 / 7}} \exp \left[-t^{2}(\xi+\mu / 2)\right] d t \\
& \leqslant \frac{\xi \exp [\Psi(\xi ; y, \mu)]}{(\xi+\mu / 2)^{1 / 2}} \cdot e^{-y^{2 / 7} / 2} .
\end{aligned}
$$

Turn to $I_{1}$. Since

$$
\Psi_{s}^{(3)}(s ; y, \mu)=-\frac{2 y}{s^{3}}=O\left(y \xi^{-3}\right)
$$

we have

$$
\Psi(s ; y, \mu)=\Psi(\xi ; y, \mu)-\frac{t^{2}}{2}\left(\mu+2 \xi+y \xi^{-2}\right)+O\left(y \xi^{-3} \mu^{-3 / 2} y^{3 / 7}\right)
$$


and

$$
y \xi^{-3} \mu^{-3 / 2} y^{3 / 7}=\frac{y^{3 / 7-1 / 2}}{\left(\frac{\mu \xi^{2}}{y}\right)^{3 / 2}} \leqslant{ }_{b} y^{-1 / 14}
$$

Consequently

$$
\begin{aligned}
I_{1} & \sim \xi \exp [\Psi(\xi ; y, \mu)] \int_{|t| \leqslant \mu^{-1 / 2} y^{1 / 7}} \exp \left(-\frac{t^{2}}{2}\left(\mu+2 \xi+y \xi^{-2}\right)\right) d t \\
& \sim \xi \exp [\Psi(\xi ; y, \mu)]\left(\frac{2 \pi}{\mu+2 \xi+y \xi^{-2}}\right)^{1 / 2} .
\end{aligned}
$$

Since $y \xi^{-2}=O(\mu),(\mathrm{A} .5)-(\mathrm{A} .6)$ imply that $I_{1} \gg I_{2}$, hence

$$
A(y, \mu) \sim e^{-\mu^{3} / 6}(2 \pi)^{-1} I_{1},
$$

which proves (2.3.23).

If we drop the condition $y \rightarrow \infty$, then the integral in (2.3.19) is of order

$$
\xi \exp [\Psi(\xi ; y, \mu)] \int_{-\infty}^{\infty} \exp \left[-t^{2}(\xi+\mu / 2)\right] d t=\xi \exp [\Psi(\xi ; y, \mu)]\left(\frac{2 \pi}{\xi+\mu / 2}\right)^{1 / 2}
$$

which proves (2.3.24).

Proof of (1.0.5). The system (1.0.1) is solvable iff for every cycle $C$ of $G$,

$$
\sum_{e \in E(C)} b_{e}=O(\bmod 2)
$$

If $b_{e} \in\{0,1\}$ are independent random variables with $\operatorname{Pr}\left(b_{e}=1\right)=\hat{p}$, the condition (A.7) is met with probability $\left(1+(1-2 \hat{p})^{|C|}\right) / 2$, Kolchin [14].

Consider $G=G(n, p=\gamma / n), \gamma<1$. Let $X_{n s}$ denote the number of cycles of length $s$ which are "bad", i. e. do not meet the condition (A.7). We need to find the limiting distribution of $X_{n}=\sum_{s \geqslant 3} X_{n s}$ the total number of "bad" cycles. To this end, observe that, with probability approaching 1 , the cycles $G(n, p)$ may have are those in the unicyclic components. Let us call them u-cycles. The expected number of all cycles of length $k \geqslant 3$ is

$$
\left(\begin{array}{l}
n \\
k
\end{array}\right) \frac{(k-1) !}{2} p^{k} \leqslant \frac{\gamma^{k}}{2 k}
$$

So

$$
\lim _{A \rightarrow \infty} \lim _{n \rightarrow \infty} \operatorname{Pr}(G(n, p) \text { has a cycle of length } \geqslant A)=0
$$


Let $Y_{n s}$ be the total number of all u-cycles of length $s$. In [20] it was proven that, for $\gamma$ fixed, $\left\{Y_{n s}\right\}_{s \leqslant A}$ converges in distribution to $\left\{\operatorname{Poisson}\left(\sigma_{s}\right)\right\}_{s \leqslant A}$, where the Poissons are independent and

$$
\sigma_{s}=\frac{T^{s}\left(\gamma e^{-\gamma}\right)}{2 s}, \quad s \geqslant 3 .
$$

As $\gamma<1$, we have $T\left(\gamma e^{-\gamma}\right)=\gamma$, because $T(x)=x e^{T(x)}$, for $x<e^{-1}$. Now a u-cycle of length $s$ is bad with probability

$$
\pi_{s}=\frac{1-(1-2 \hat{p})^{s}}{2} .
$$

Consequently $\left\{X_{n s}\right\}_{s \leqslant A}$ converges to $\left\{\operatorname{Poisson}\left(\pi_{s} \sigma_{s}\right)\right\}_{s \leqslant A}$, whence $X_{n}$ converges to Poisson $\left(\sum_{s \leqslant A} \pi_{s} \sigma_{s}\right)$. Therefore,

$$
\lim _{n \rightarrow \infty} \operatorname{Pr}\{\text { there are nod bad u-cycles of length } A \text { at most }\}=e^{-\sum_{s \leqslant A} \pi_{s} \sigma_{s}} .
$$

It remains to notice that

$$
\begin{aligned}
\sum_{s \geqslant 3} \pi_{s} \sigma_{s} & =\sum_{s \geqslant 3} \frac{1-(1-2 \hat{p})^{s}}{2} \frac{\gamma^{s}}{2 s} \\
& =\frac{1}{4} \ln \frac{1-\gamma(1-2 \hat{p})}{1-\gamma}-\frac{\gamma}{2} \hat{p}-\frac{\gamma^{2}}{2} \hat{p}(1-\hat{p}) .
\end{aligned}
$$

That the same formula holds for $G(n, m=\gamma n / 2)$ follows then in a standard way. 